\title{
Analysis of a moving mask approximation for martensitic transformations
}

\author{
Francesco Della Porta \\ Mathematical Institute, University of Oxford \\ Oxford OX2 6GG, UK \\ francesco.dellaporta@maths.ox.ac.uk*
}

November 21, 2018

\begin{abstract}
In this work we introduce a moving mask approximation to describe the dynamics of austenite to martensite phase transitions at a continuum level. In this framework, we prove a new type of Hadamard jump condition, from which we deduce that the deformation gradient must be of the form $1+\mathbf{a} \otimes \mathbf{n}$ a.e. in the martensite phase. This is useful to better understand the complex microstructures and the formation of curved interfaces between phases in new ultra-low hysteresis alloys such as $\mathrm{Zn}_{45} \mathrm{Au}_{30} \mathrm{Cu}_{25}$, and provides a selection mechanism for physically-relevant energyminimising microstructures. In particular, we use the new type of Hadamard jump condition to deduce a rigidity theorem for the two well problem. The latter provides more insight on the cofactor conditions, particular conditions of supercompatibility between phases believed to influence reversibility of martensitic transformations.
\end{abstract}

\section{Introduction}

The aim of this work is to study from a mathematical point of view the complex microstructures arising during the austenite to martensite phase transition in ultra-low hysteresis alloys such as $\mathrm{Zn}_{45} \mathrm{Au}_{30} \mathrm{Cu}_{25}$ (see [31]). Austenite to martensite transitions are solid to solid phase transitions, in which the underlying crystal lattice of an alloy experiences a change of shape as temperature is moved across a certain critical temperature $\theta_{T}$. When the temperature is above $\theta_{T}$, the alloy has a unique crystalline structure, called austenite, which is energetically preferable; when the temperature is lowered below $\theta_{T}$, the energetically preferable state for the crystal is no longer austenite but martensite, which usually has more then one variant. Often, a change of crystalline structure implies a change in the macroscopic properties of the material, which can thus be controlled by changing the temperature of the sample. A serious obstacle to practical applications of shape-memory and other such materials

\footnotetext{
${ }^{*}$ Current institution: Max-Planck Institute for Mathematics in the Sciences, Leipzig, Germany, Francesco.DellaPorta@mis.mpg.de

Acknowledgements: This work was supported by the Engineering and Physical Sciences Research Council [EP/L015811/1]. The author would like to thank John Ball for his helpful suggestions and feedback which greatly improved this work, as well as Richard James, Giacomo Canevari and Xian Chen for the useful discussions.
} 
is reversibility of the transformation. Indeed, after a small number of cycles, one can usually observe a shift in the transition temperature and in the latent heat. Furthermore, the formation of micro-cracks during the phase transition often leads to early failure by rupture.

An important step towards understanding the factors influencing reversibility can be found in 12]. There, the authors study particular conditions of geometric compatibility between martensitic variants called cofactor conditions, that were first introduced in [6]. Among these conditions, there is the requirement that the middle eigenvalue of the lattice transformation matrices is equal to 1 , which was previously shown (see [33]) to influence reversibility. In [12], the authors prove that under the cofactor conditions no elastic transition layer is needed to make simple laminates compatible with austenite, and point out that this fact might have important consequences on the reversibility of the phase transitions. Indeed, the authors observe that transition layers are intuitively both a cause of thermal hysteresis, and of the formation of dislocations and nucleation of micro-cracks, that, after many cycles, induce loss of good reversibility properties.

The recent fabrication announced in 31] of $\mathrm{Zn}_{45} \mathrm{Au}_{30} \mathrm{Cu}_{25}$, the first material closely satisfying the cofactor conditions (the relative error is of order $10^{-4}$ ), partially confirms this conjecture. Indeed, this material exhibits ultra-low hysteresis and does not seem to incur any loss of reversibility after more than 16,000 thermal cycles. We refer the reader interested in ultra-low hysteresis alloys also to [14], where the fabrication of a new material undergoing $10^{7}$ cycles with very little fatigue is announced. A discussion on the relation between the cofactor conditions and ultra-low hysteresis alloys can be found in [26, 27].

As remarked in [31], it is intriguing and unusual that martensitic microstructures in $\mathrm{Zn}_{45} \mathrm{Au}_{30} \mathrm{Cu}_{25}$ are drastically different in consecutive thermally induced transformation cycles, this being partially motivated by the fact that the cofactor conditions are close to being satisfied by both some type I and some type II twins, which can all form zero energy interfaces with austenite.

The aim of this paper is to further study these microstructures, and to identify a common characterization for all of them. To this end we start from the following observation: from the dynamical point of view, it looks as if in every thermally induced phase transformation cycle in $\mathrm{Zn}_{45} \mathrm{Au}_{30} \mathrm{Cu}_{25}$ there was a mask moving across the domain covering and uncovering martensite microstructures. This is equivalent to saying that the martensitic microstructures do not change after the phase transition has happened, which seems to be a particularly legitimate approximation in materials satisfying the cofactor conditions, where no interface layer is needed between phases. But this hypothesis makes sense also in many other materials as long as one considers macroscopic deformations.

As a first step, we give a mathematical characterisation of the moving mask approximation and we frame it in the context of nonlinear elasticity, where phase changes are interpreted as elastic deformations. In particular, microstructures satisfying the moving mask approximation are special solutions to a simplified model for the dynamics of martensitic transformations which was introduced in [15]. The model in [15] is derived from the equations for the conservation of energy and momentum in the context of dynamics for nonlinear elasticity, and describes the evolution of the phase interface as a moving shock wave (see Section 3). Then, by deriving a new type of Hadamard jump condition (Section 4), we prove that every martensitic microstructure satisfying the moving mask assumption and some further technical hypotheses must be of the form

$$
\nabla \mathbf{y}(\mathbf{x})=1+\mathbf{a}(\mathbf{x}) \otimes \mathbf{n}(\mathbf{x}), \quad \text { a.e., }
$$

where $\mathbf{n}(\mathbf{x})$ is, up to a change of sign, the phase interface normal at $\mathbf{x}$ when the point $\mathbf{x}$ is on the 
interface. The above result does not follow directly from the assumption that the deformation gradient is unchanged after the phase transition, and is not a direct consequence of previously known Hadamard jump conditions if $\mathbf{y}$ is just Lipschitz as in our case. We refer the interested reader to Section 2.1 for a brief review on known versions of the Hadamard jump conditions and on why they do not imply (1.1). Subsequent experiments (see [13]) have measured $\|\operatorname{cof}(\nabla \mathbf{y}-1)\|$ in a sample of $\mathrm{Zn}_{45} \mathrm{Au}_{30} \mathrm{Cu}_{25}$. The measured values are of the order of $10^{-4}$, which seem to be small enough to partially confirm the validity of (1.1) and of the moving mask approximation. Conversely, from the moving mask hypotheses and our new Hadamard jump condition, we can reconstruct the position of the austenite-martensite interface during the phase transition from a martensitic deformation gradient.

Under suitable hypotheses, we prove also that $\nabla \cdot \mathbf{a}=0$. This result relies on a Hadamard jump condition for strains in $B V(\Omega)$ that is proved in Section 5 and generalizes that in [18]. As a consequence of the fact that $\nabla \mathbf{y}=1+\mathbf{a} \otimes \mathbf{n}$ almost everywhere in the martensitic microstructures, we can prove a rigidity theorem for compound twins and a result that allows for a better understanding of the nature of curved austenite-martensite interfaces for type I twins. Under some further assumption, we can extend the rigidity result to a general two well problem not satisfying the cofactor conditions. This result explains the importance of satisfying the cofactor conditions in order to have non-constant average deformation gradients of the form (1.1), which are obtained by finely mixing two martensitic variants.

The dynamics of the phase transition in $\mathrm{Zn}_{45} \mathrm{Au}_{30} \mathrm{Cu}_{25}$ are very complex, and far from being completely understood. As in the static case, a major obstacle towards a good understanding of the phenomenon remains the lack of a characterization of the quasiconvex hull of the set of possible deformation gradients. Nonetheless, our results provide an interesting set of tools that can be used to understand further the complex microstructures arising in martensitic phase transitions. Indeed, our moving mask hypothesis can be seen as a selection mechanism for physicaly relevant energy minimising microstructures arising in thermally induced martensitic transformations.

Further investigation on why martensitic microstructures are so different in different thermal cycles in $\mathrm{Zn}_{45} \mathrm{Au}_{30} \mathrm{Cu}_{25}$ is carried out in [16]. Indeed, in [16] we show that this material satisfies some further conditions of compatibility (on top of the cofactor conditions) that makes the set of possible macroscopic deformation gradients of the form (1.1) unusually large.

The plan for the paper is the following: in Section 2.1 we give a brief overview of the nonlinear elasticity model, and introduce concepts which will be useful for our analysis, namely twinning, the cofactor conditions and k-rectifiable sets. In Section 3 we recall results from [15] and in this context we introduce the mathematical definition of moving mask approximation. In Section 4 we prove a dynamic variant of the Hadamard jump condition for Lipschitz functions and curved interfaces. As explained above, the results rely on the hypothesis that the deformation gradient remains constant in time at a point of the domain, once the phase transition has occurred. The last two sections are devoted to proving the rigidity results, and some results on moving austenite-martensite interfaces and on possible microstructures that can be explained using our model. 


\section{Preliminaries}

\subsection{Nonlinear elasticity model}

In order to describe austenite to martensite phase transitions in crystalline solids, one of the most successful mathematical continuum models is nonlinear elasticity, which has proved to capture many aspects of the physical phenomena such as the formation of twins (see [6]) and to be useful in understanding related behaviour such as the shape-memory effect (see [10]), and, more recently, hysteresis (see [33]). In this and the next subsection, we give a brief overview of the theory following closely [9, 12]. For more details we refer the reader to $[6,8,11]$.

The nonlinear elasticity model is based on the idea of looking at changes in the crystal lattice as elastic deformations in the continuum mechanics framework. Following [6], we hence assume that the deformations minimize a free energy of the type

$$
\mathcal{E}(\mathbf{y}, \theta)=\int_{\Omega} \phi(\nabla \mathbf{y}(\mathbf{x}), \theta) \mathrm{d} \mathbf{x} .
$$

Here, $\theta$ denotes the temperature of the crystal. Three different regimes are distinguishable depending on this parameter: $\theta<\theta_{T}$ and $\theta>\theta_{T}$, where respectively martensite and austenite phases minimize the energy, and $\theta=\theta_{T}$ where these are energetically equivalent. In (2.2), the bounded Lipschitz domain (open and connected) $\Omega$ stands for the reference configuration of undistorted austenite at $\theta=\theta_{T}$ and $\mathbf{y}(\mathbf{x})$ denotes the position of the particle $\mathbf{x} \in \Omega$ after the deformation. Finally, $\phi$ is the free-energy density, depending on the temperature $\theta$ and the deformation gradient $\nabla \mathbf{y}$, satisfying the following properties:

- $\mathcal{D}:=\left\{\mathrm{F} \in \mathbb{R}^{3 \times 3}: \operatorname{det} \mathrm{F}>0\right\}$,

$$
\phi(\cdot, \theta): \mathcal{D} \rightarrow \mathbb{R}
$$

is a function bounded below by a constant depending on $\theta$ for each $\theta>0$;

- $\phi(\cdot, \theta)$ satisfies frame-indifference, i.e., for all $\mathrm{F} \in \mathcal{D}$ and all rotations $\mathrm{R} \in S O(3), \phi(\mathrm{RF}, \theta)=$ $\phi(\mathrm{F}, \theta)$. This property reflects the invariance of the free-energy density under rotations;

- $\phi$ has cubic symmetry, i.e., $\phi(\mathrm{FQ}, \theta)=\phi(\mathrm{F}, \theta)$ for all $\mathrm{F} \in \mathcal{D}$ and all rotations $\mathrm{Q}$ in the symmetry group of austenite $\mathcal{P}^{24}$, the group of rotations sending a cube into itself (see [11] for more details);

- denoting by $K_{\theta}$ the set of minima for the free-energy density at temperature $\theta$, i.e., $K_{\theta}:=\{\mathrm{F} \in$ $\mathcal{D}: \mathrm{F} \in \operatorname{argmin}(\phi(G, \theta))\}$,

$$
K_{\theta}= \begin{cases}\alpha(\theta) S O(3), & \theta>\theta_{T} \\ S O(3) \cup \bigcup_{i=1}^{N} S O(3) \cup_{i}\left(\theta_{T}\right), & \theta=\theta_{T} \\ \bigcup_{i=1}^{N} S O(3) \bigcup_{i}(\theta), & \theta<\theta_{T} .\end{cases}
$$

Here, $\alpha(\theta)$ is a scalar dilatation coefficient satisfying $\alpha\left(\theta_{T}\right)=1$, while $\mathbf{U}_{i}(\theta) \in \mathbb{R}_{\text {Sym }^{+}}^{3 \times 3}$ are the $N$ positive definite symmetric matrices corresponding to the transformation from austenite to the $N$ variants of martensite at temperature $\theta$. Here and below $\mathbb{R}_{S y m^{+}}^{3 \times 3}$ represents the set of $3 \times 3$ symmetric and positive definite matrices. From now on, we omit the dependence on the temperature in $K_{\theta}$ when $\theta<\theta_{T}$, and neglect the dependence on $\theta$ of the $\mathrm{U}_{i}$. We remark that for each $\mathrm{U}_{i}, \mathrm{U}_{j}$ there exists $\mathrm{R} \in \mathcal{P}^{24}$ such that $\mathrm{R}^{T} \mathrm{U}_{j} \mathrm{R}=\mathrm{U}_{i}$, so that $\mathrm{U}_{i}, \mathrm{U}_{j}$ share the same eigenvalues. 
Based on both experimental evidence and the mathematical complexity of other cases, most results in the literature are related to planar austenite-martensite interfaces $\left\{\mathbf{x} \in \mathbb{R}^{3}: \mathbf{x} \cdot \mathbf{n}=k\right\}$, with normal $\mathbf{n}$, at $\theta=\theta_{T}$. In this case, under suitable conditions on the lattice deformation and for some $\mathbf{n} \in \mathbb{R}^{3}$, it is possible to construct a sequence $\mathbf{y}^{j}$ such that

$$
\begin{array}{lll}
\nabla \mathbf{y}^{j} \rightarrow S O(3) & \text { in measure } & \text { for } \mathbf{x} \cdot \mathbf{n}<k \\
\nabla \mathbf{y}^{j} \rightarrow \bigcup_{i=1}^{N} S O(3) \mathbf{U}_{i} & \text { in measure } & \text { for } \mathbf{x} \cdot \mathbf{n}>k .
\end{array}
$$

Denoting by $\mathscr{L}^{3}$ the three-dimensional Lebesgue measure, we notice that (2.4)-(2.5) imply

$$
\lim _{j \rightarrow \infty} \mathscr{L}^{3}\left\{\mathbf{x} \in \Omega: \nabla \mathbf{y}^{j}(\mathbf{x}) \notin K_{\theta_{T}}\right\}=0,
$$

and, under some further hypotheses on $\phi, \mathbf{y}^{j}$ is a minimizing sequence for $\mathcal{E}\left(\cdot, \theta_{T}\right.$ ) (see [6] for more details). Furthermore, since $\mathbf{y}^{j}$ can be constructed so as to be bounded in $W^{1, \infty}\left(\Omega, \mathbb{R}^{3}\right)$, there exists a subsequence $\mathbf{y}^{k_{j}}$ and $\mathbf{y} \in W^{1, \infty}\left(\Omega, \mathbb{R}^{3}\right)$ such that $\mathbf{y}^{k_{j}}$ converges to $\mathbf{y}$ weakly* in the same space. However, the energy functional is not quasiconvex and, in general, the minimum is not attained in the classical sense. Therefore, $\nabla \mathbf{y}$ is not a minimizer for $\mathcal{E}\left(\cdot, \theta_{T}\right)$, but just of its relaxation, $\mathcal{E}^{q c}\left(\cdot, \theta_{T}\right)$. From a physical point of view, $\nabla \mathbf{y}$ represents the deformation gradient in the sample at a macroscopic scale, an average of the fine microstructures with gradients in

$$
K:=\bigcup_{i=1}^{N} S O(3) \mathrm{U}_{i}
$$

It is important to remark that, in general, macroscopic deformation gradients $\nabla \mathbf{y}$ are not elements of $K$ a.e. in $\Omega$. Instead, we have $\nabla \mathbf{y} \in K^{q c}$ a.e. in $\Omega$, where

$$
K^{q c}:=\left\{\mathrm{M} \in \mathbb{R}^{3 \times 3} \mid \begin{array}{c}
f(\mathrm{M}) \leq \max _{K} f, \text { for all continuous } \\
\text { quasiconvex } f: \mathbb{R}^{3 \times 3} \rightarrow \mathbb{R}
\end{array}\right\},
$$

is the quasiconvex hull of the set $K$ (see [29]). Characterizing the set of possible macroscopic deformations $K^{q c}$ is very important in order to fully understand the nonlinear elasticity model. On the other hand, the set of constant macroscopic gradients B which can form an interface with austenite, having constant gradient $\mathrm{A}$, is in general smaller then the whole of $K^{q c}$. Indeed, a Lipschitz function whose gradient is equal to $A, B$ a.e. in $\Omega$, with $A, B \in \mathbb{R}^{3 \times 3}$ must satisfy a generalized version of the Hadamard jump condition proved in [6] :

Proposition 2.1 ([6, Prop. 1]). Let $\Omega \in \mathbb{R}^{3}$ be open and connected. Assume $\mathbf{y} \in W^{1, \infty}\left(\Omega, \mathbb{R}^{3}\right)$ satisfies

$$
\nabla \mathbf{y}(\mathbf{x})=\mathrm{A}, \quad \text { a.e. } \mathbf{x} \in \Omega_{A} ; \quad \nabla \mathbf{y}(\mathbf{x})=\mathrm{B}, \quad \text { a.e. } \mathbf{x} \in \Omega_{B},
$$

where $\mathrm{A}, \mathrm{B} \in \mathbb{R}^{3 \times 3}$ and $\Omega_{A}, \Omega_{B}$ are disjoint measurable sets such that

$$
\Omega_{A} \cup \Omega_{B}=\Omega, \quad \mathscr{L}^{3}\left(\Omega_{A}\right)>0, \quad \mathscr{L}^{3}\left(\Omega_{B}\right)>0 .
$$

Then,

$$
\mathrm{A}-\mathrm{B}=\mathbf{a} \otimes \mathbf{n}, \quad \mathbf{a}, \mathbf{n} \in \mathbb{R}^{3},|\mathbf{n}|=1 .
$$


This result forces a fixed macroscopic gradient of martensite with constant gradient $B$ to be rankone connected to austenite, having constant gradient A, across every interface between the two. Furthermore, it implies that, in this case, every phase interface is planar. For these reasons, Proposition 2.1 is the background for many results for compatibility between phases.

However, this type of result fails to be true for more general gradients $\nabla \mathbf{y} \in L^{\infty}\left(\Omega ; \mathbb{R}^{3 \times 3}\right)$. As a matter of fact, as shown in [6], it is possible to construct a Lipschitz function $\mathbf{z} \in W^{1, \infty}\left(\Omega, \mathbb{R}^{3}\right)$ which is constant in the set $\Omega \cap\{\mathbf{x}: \mathbf{x} \cdot \mathbf{n}>c\}$ for some $c \in \mathbb{R}, \mathbf{n} \in \mathbb{R}^{3}$, and whose gradient $\nabla \mathbf{y} \in\left\{\mathrm{F}_{1}, \mathrm{~F}_{2}, \mathrm{~F}_{3}, \mathrm{~F}_{4}\right\}$ in $\Omega \cap\{\mathbf{x}: \mathbf{x} \cdot \mathbf{n}<c\}$ for some matrices $\mathrm{F}_{i}$, such that $\nabla \mathbf{y}$ is not rank-one almost everywhere in $\Omega \cap\{\mathbf{x}: \mathbf{x} \cdot \mathbf{n}<c\}$. Indeed a fractal behaviour of $\nabla \mathbf{y}$ close to $\mathbf{x} \cdot \mathbf{n}=c$, finely mixing martensitic variants near the interface, allows one to achieve compatibility between incompatible gradients. That is, compatibility is achieved on the average. Possible approaches to recovering the Hadamard jump condition in an average sense can be found in [3], or in Remark 5.1 below. Another generalization of Proposition 2.1 was proved in [9] by assuming $\mathbf{y} \in W^{1, \infty}\left(\Omega, \mathbb{R}^{3}\right)$ to be $C^{1}$ both in $\bar{\Omega}_{A}$ and $\bar{\Omega}_{B}$, with $\Omega_{A}, \Omega_{B}$ two open disjoint subdomains of $\Omega$, separated by a piecewise $C^{1}$, possibly curved, 2-dimensional interface $\Gamma$ such that $\Omega=\Omega_{A} \cup \Omega_{B} \cup \Gamma$. In the case of martensitic transformations, $\nabla \mathbf{y}=1$ in $\Omega_{A}$, while in $\Omega_{B} \nabla \mathbf{y}$ represents a continuously varying macroscopic deformation gradient corresponding to a continuously varying martensitic microstructure. This result can be extended to $\mathbf{y} \in H^{1}\left(\Omega, \mathbb{R}^{3}\right)$ with $\nabla \mathbf{y} \in B V\left(\Omega ; \mathbb{R}^{3 \times 3}\right)$ as done in the two dimensional setting in [18], or more generally in Lemma 5.1 below. However, the deep result of [25] states that, in the case where $\mathbf{y} \in W^{1, \infty}\left(\mathbb{R}^{3}, \mathbb{R}^{3}\right)$, and $\mathbf{y}$ is constant in $\{\mathbf{x} \cdot \mathbf{n}>c\}$, the polyconvex hull of the set $\{\nabla \mathbf{y}(\mathbf{x}): \mathbf{x} \in\{\mathbf{x} \cdot \mathbf{n}<c\}\}$, which contains $\{\nabla \mathbf{y}(\mathbf{x}): \mathbf{x} \in\{\mathbf{x} \cdot \mathbf{n}<c\}\}^{q c}$, might not contain a matrix which is rank-one connected to 0 , the deformation gradient in $\{\mathbf{x} \cdot \mathbf{n}>c\}$.

On the other hand, in order to fully capture the complex microstructures observed in $\mathrm{Zn}_{45} \mathrm{Au}_{30} \mathrm{Cu}_{25}$, we are interested in macroscopic deformation gradients that are just in $L^{\infty}\left(\Omega, \mathbb{R}^{3 \times 3}\right)$. Therefore, in Section 4 we generalize Proposition 2.1 to non-constant deformation gradients in $L^{\infty}\left(\Omega ; \mathbb{R}^{3 \times 3}\right)$ and to curved interfaces. However, given the above mentioned counterexamples of [6, 25], we need to change perspective and introduce some further hypotheses. This is done by recalling the idea of a moving mask explained in the introduction, which is mathematically framed in Section 3, and where the deformation gradient at a certain point $\mathbf{x}$ changes only once during phase transition, i.e., when the martensite-austenite interface passes through $\mathbf{x}$.

\subsection{Twinning theory and the cofactor conditions}

As explained in the previous section, the existence of a constant macroscopic martensitic deformation compatible with austenite, is related to the existence of a matrix $\mathrm{F} \in K^{q c}$ such that $\mathrm{F}=\mathbf{1}+\mathbf{a} \otimes \mathbf{n}$. Conditions on the deformation parameters under which such matrices exist have been first investigated in [6] in the case of two wells, i.e., $N=2$, and then generalized in [4, 5]. The case where $N=2$ is the most widely studied, as it is the only one for which an explicit characterization of $K^{q c}$ is known, and turns out to be a fundamental tool to explain a wide range of experimental observations. Therefore, we now focus on the possibility of a pair of martensitic variants forming interfaces with austenite. The notation and results of this section follow closely those in [12].

Let us first recall that given two different variants of martensite, represented by $\mathrm{U}_{1}, \mathrm{U}_{2} \in \mathbb{R}_{S y m^{+}}^{3 \times 3}$, there exists a rotation $\mathrm{R} \in S O(3)$ satisfying $\mathrm{U}_{2}=\mathrm{RU}_{1} \mathrm{R}^{T}$. A first useful result is the following: 
Proposition 2.2 ([12, Prop. 12]). Let $\mathrm{U}_{1}, \mathrm{U}_{2} \in \mathbb{R}_{S y m^{+}}^{3 \times 3}$ with $\mathrm{U}_{1} \neq \mathrm{U}_{2}$. Suppose further that they are compatible in the sense that there is a matrix $\hat{\mathrm{R}} \in S O(3)$ such that

$$
\hat{\mathrm{R}} \mathrm{U}_{2}-\mathrm{U}_{1}=\mathbf{b} \otimes \mathbf{m}
$$

$\mathbf{b}, \mathbf{m} \in \mathbb{R}^{3}$. Then there is a unit vector $\hat{\mathbf{e}} \in \mathbb{R}^{3}$ such that

$$
\mathrm{U}_{2}=(-1+2 \hat{\mathbf{e}} \otimes \hat{\mathbf{e}}) \mathrm{U}_{1}(-1+2 \hat{\mathbf{e}} \otimes \hat{\mathbf{e}}) .
$$

Conversely, if (2.7) is satisfied, then there exist $\hat{\mathrm{R}} \in S O(3), \mathbf{b}, \mathbf{m} \in \mathbb{R}^{3}$ such that (2.6) holds.

Equation (2.6) is called the compatibility condition for two variants of martensite; the solutions to this equation can be classified into three categories: compound, type I and type II twins. It is possible to prove that (see e.g., [11]), once $U_{1}$ and $U_{2}$ are given and (2.7) holds, the compatibility condition always has two solutions $\left(\hat{\mathrm{R}}_{I}, \mathbf{b}_{I} \otimes \mathbf{m}_{I}\right)$ and $\left(\hat{\mathrm{R}}_{I I}, \mathbf{b}_{I I} \otimes \mathbf{m}_{I I}\right)$. The solutions can be expressed as follows:

$$
\begin{array}{lll}
\text { type I } & \mathbf{m}_{I}=\hat{\mathbf{e}}, & \mathbf{b}_{I}=2\left(\frac{\mathrm{U}_{1}^{-1} \hat{\mathbf{e}}}{\left|\mathrm{U}_{1}^{-1} \hat{\mathbf{e}}\right|^{2}}-\mathrm{U}_{1} \hat{\mathbf{e}}\right), \\
\text { type II } & \mathbf{m}_{I I}=2\left(\hat{\mathbf{e}}-\frac{\mathrm{U}_{1}^{2} \hat{\mathbf{e}}}{\left|\mathrm{U}_{1} \hat{\mathbf{e}}\right|^{2}}\right), & \mathbf{b}_{I I}=\mathrm{U}_{1} \hat{\mathbf{e}},
\end{array}
$$

where $\hat{\mathbf{e}}$ is as in (2.7). If ê satisfying (2.7) is unique up to change of sign, the two solutions (2.8) and (2.9) of (2.6) are called type I and type II twins respectively. In case there exist two different nonparallel unit vectors satisfying (2.7), the resulting pair of solutions (2.8)-(2.9) are called compound twins. Nonetheless, it is possible to prove (see e.g., 11]) that in the case of compound twins, given two different unit vectors satisfying (2.7), namely $\hat{\mathbf{e}}_{1}$ and $\hat{\mathbf{e}}_{2}$, then

$$
\mathbf{b}_{I}^{1} \otimes \mathbf{m}_{I}^{1}:=2\left(\frac{\mathrm{U}_{1}^{-1} \hat{\mathbf{e}}_{1}}{\left|\mathrm{U}_{1}^{-1} \hat{\mathbf{e}}_{1}\right|^{2}}-\mathrm{U}_{1} \hat{\mathbf{e}}_{1}\right) \otimes \hat{\mathbf{e}}_{1}=\mathrm{U}_{1} \hat{\mathbf{e}}_{2} \otimes 2\left(\hat{\mathbf{e}}_{2}-\frac{\mathrm{U}_{1}^{2} \hat{\mathbf{e}}_{2}}{\left|\mathrm{U}_{1} \hat{\mathbf{e}}_{2}\right|^{2}}\right)=: \mathbf{b}_{I I}^{2} \otimes \mathbf{m}_{I I}^{2} .
$$

Therefore, there are just two solutions to (2.7), even in the case of compound twins, each of which can be considered as both a type I and a type II twin. Below, however, when we refer to type I or type II solutions of (2.6) we assume implicitly that they are not compound solutions. Furthermore, we sometimes abuse of notation and write that $\mathrm{U}_{1}, \mathrm{U}_{2}$ form a compound twin if the solutions of the twinning equations (2.6) are compound twins. The following characterization of compound twins is used below:

Proposition 2.3 ([12, Prop. 1]). Let $\mathrm{U}_{1}$ and $\mathrm{U}_{2}$ be two different variants of martensite and $\hat{\mathbf{e}}_{1}$ a unit vector such that (2.7) is satisfied. Then there exists a second unit vector $\hat{\mathbf{e}}_{2}$ not parallel to $\hat{\mathbf{e}}_{1}$ satisfying (2.7) if and only if $\hat{\mathbf{e}}_{1}$ is perpendicular to an eigenvector of $\mathrm{U}_{1}$. When this condition is verified, $\hat{\mathbf{e}}_{2}$ is unique up to change of sign and is perpendicular to both $\hat{\mathbf{e}}_{1}$ and that eigenvector.

Let us now consider a simple laminate, i.e., a constant macroscopic gradient $\nabla \mathbf{y}$ equal a.e. to $\lambda \hat{\mathrm{R}} \mathrm{U}_{2}+(1-\lambda) \mathrm{U}_{1}$ for some $\lambda \in(0,1)$ and some rank-one connected $\mathrm{RU}_{2}, \mathrm{U}_{1} \in K$. Following [6, 12] we focus on the possibility for such $\nabla \mathbf{y}$ to be compatible with austenite. By Proposition [2.1, a necessary condition is that $S O(3)$ has a rank-one connection with $\lambda \hat{\mathrm{R}} \mathrm{U}_{2}+(1-\lambda) \mathrm{U}_{1}$. The existence of $(\mathrm{R}, \lambda, \mathbf{a} \otimes \mathbf{n})$ solving

$$
\mathrm{R}\left[\lambda \hat{\mathrm{R}} \mathrm{U}_{2}+(1-\lambda) \mathrm{U}_{1}\right]-1=\mathrm{R}\left[\lambda\left(\mathrm{U}_{1}+\mathbf{b} \otimes \mathbf{m}\right)+(1-\lambda) \mathrm{U}_{1}\right]-1=\mathbf{a} \otimes \mathbf{n}
$$


that is a twinned laminate compatible with austenite, was first studied in [32] and later in [6]. Lattice deformations and parameters of materials that are usually considered in the literature lead to twins with exactly four solutions to equation (2.10). Nonetheless, in some cases the number of solutions can be just zero, one or two, and, under some particular condition on the lattice parameters, as in the case of the material discovered in [31], (2.10) is satisfied for all $\lambda \in[0,1]$. The following result gives necessary and sufficient conditions for this to hold:

Theorem 2.1 ([12, Thm. 2]). Let $\mathrm{U}_{1}, \mathrm{U}_{2} \in \mathbb{R}_{\text {Sym }}^{3 \times 3}$ be distinct and such that there exist $\hat{\mathrm{R}} \in S O(3)$ and $\mathbf{b}, \mathbf{m} \in \mathbb{R}^{3}$ satisfying

$$
\hat{\mathrm{R}} \mathrm{U}_{2}=\mathrm{U}_{1}+\mathbf{b} \otimes \mathbf{m}
$$

Then, (2.10) has a solution $\mathrm{R} \in S O(3)$, $\mathbf{a}, \mathbf{n} \in \mathbb{R}^{3}$ for each $\lambda \in[0,1]$ if and only if the following cofactor conditions hold:

(CC1) The middle eigenvalue $\lambda_{2}$ of $\mathrm{U}_{1}$ satisfies $\lambda_{2}=1$,

(CC2) b $\cdot \mathrm{U}_{1} \operatorname{cof}\left(\mathrm{U}_{1}^{2}-1\right) \mathbf{m}=0$,

(CC3) $\operatorname{tr} U_{1}^{2}-\operatorname{det} U_{1}^{2}-\frac{1}{4}|\mathbf{b}|^{2}|\mathbf{m}|^{2}-2 \geq 0$.

In the last part of this section, we report some results from [12] related to the cofactor conditions in type I/II twins.

Theorem 2.2 ([12, Thm. 7]). Let $\mathrm{U}_{1}, \mathrm{U}_{2} \in \mathbb{R}_{\text {Sym }}^{3 \times 3}$ be distinct and such that $\hat{\mathrm{R}} \in S O(3), \mathbf{b}_{I}, \mathbf{m}_{I} \in \mathbb{R}^{3}$ is a type I solution to (2.6). Suppose further that $\mathrm{U}_{1}, \mathbf{b}_{I}, \mathbf{m}_{I}$ satisfy the cofactor conditions. Then, there exist $\mathrm{R}_{0} \in S O(3), \mathbf{a}_{0} \in \mathbb{R}^{3}, \mathbf{n}_{0}, \mathbf{n}_{1} \in \mathbb{S}^{2}$ and $\xi \neq 0$ such that

$$
\mathrm{R}_{0} \mathrm{U}_{1}=1+\mathbf{a}_{0} \otimes \mathbf{n}_{0}, \quad \mathrm{R}_{0}\left(\mathrm{U}_{1}+\mathbf{b}_{I} \otimes \mathbf{m}_{I}\right)=1+\mathbf{a}_{0} \otimes \xi \mathbf{n}_{1} .
$$

Furthermore,

$$
\mathrm{R}_{0}\left[\mathrm{U}_{1}+\lambda \mathbf{b}_{I} \otimes \mathbf{m}_{I}\right]=1+\mathbf{a}_{0} \otimes\left(\lambda \xi \mathbf{n}_{1}+(1-\lambda) \mathbf{n}_{0}\right), \quad \text { for all } \lambda \in[0,1] .
$$

Theorem 2.3 ([12, Thm. 8]). Let $\mathrm{U}_{1}, \mathrm{U}_{2} \in \mathbb{R}_{S y m^{+}}^{3 \times 3}$ be distinct and such that $\hat{\mathrm{R}} \in S O(3), \mathbf{b}_{I I}, \mathbf{m}_{I I} \in \mathbb{R}^{3}$ is a type II solution to (2.6). Suppose further that $\mathrm{U}_{1}, \mathbf{b}_{I I}, \mathbf{m}_{I I}$ satisfy the cofactor conditions. Then, there exist $\mathrm{R}_{0} \in S O(3), \mathbf{a}_{0}, \mathbf{a}_{1} \in \mathbb{R}^{3}, \mathbf{n}_{0} \in \mathbb{S}^{2}$ and $\xi \neq 0$ such that

$$
\mathrm{R}_{0} \mathrm{U}_{1}=1+\mathbf{a}_{0} \otimes \mathbf{n}_{0}, \quad \mathrm{R}_{0}\left(\mathrm{U}_{1}+\mathbf{b}_{I I} \otimes \mathbf{m}_{I I}\right)=1+\xi \mathbf{a}_{1} \otimes \mathbf{n}_{0} .
$$

Furthermore,

$$
\mathrm{R}_{0}\left[\mathrm{U}_{1}+\lambda \mathbf{b}_{I I} \otimes \mathbf{m}_{I I}\right]=1+\left(\lambda \xi \mathbf{a}_{1}+(1-\lambda) \mathbf{a}_{0}\right) \otimes \mathbf{n}_{0}, \quad \text { for all } \lambda \in[0,1]
$$

\subsection{Some preliminaries on $k$-rectifiable sets}

In this section we recall some standard results on Lipschitz functions and $k$-rectifiable sets from [2, 21, 28] (see also [1] for properties of level sets of Lipschitz functions). We denote by $\mathscr{H}^{k}$ the $k$-dimensional Hausdorff measure, and write $\mathscr{H}^{k} \mathrm{~L} E$ for its restriction to an $\mathscr{H}^{k}$ measurable subset E. $C_{c}\left(\mathbb{R}^{d}\right)$ stands for the space of continuous functions with compact support in $\mathbb{R}^{d}$, while $B^{d}(\mathbf{x}, r)$ denotes the $d$-dimensional ball centred at $x$, with radius $r$ and of volume $\omega_{d} r^{d}$. We start with the following definitions: 
Definition 2.1. A Lipschitz $k$-graph $\mathcal{G}$ is a set of points in $\mathbb{R}^{d}$ with $d>k$ such that there exists an open and connected set $\omega \subset \mathbb{R}^{k}$, a Lipschitz map $\psi: \omega \rightarrow \mathbb{R}^{d-k}$, and a rotation $\mathrm{Q} \in S O(d)$ satisfying

$$
\mathcal{G}:=\left\{\mathbf{Q x}, \mathbf{x}=\left(\mathbf{x}^{\prime}, \boldsymbol{\psi}\left(\mathbf{x}^{\prime}\right)\right), \mathbf{x}^{\prime} \in \omega\right\} .
$$

Definition 2.2. Let $E \subset \mathbb{R}^{d}$ be an $\mathscr{H}^{k}$-measurable set satisfying $\mathscr{H}^{k}(E)<\infty$. We say that $E$ is $k$-rectifiable if there exist countably many Lipschitz mappings $\mathbf{f}_{i}: \mathbb{R}^{k} \rightarrow \mathbb{R}^{d}$ such that

$$
\mathscr{H}^{k}\left(E \backslash \bigcup_{i=1}^{\infty} \mathbf{f}_{i}\left(\mathbb{R}^{k}\right)\right)=0 .
$$

An equivalent characterization for such sets is given by the following result:

Proposition 2.4 ([2, Prop. 2.76]). Any $\mathscr{H}^{k}$-measurable set $E$ is countably $\mathscr{H}^{k}$-rectifiable if and only if there exist countably many Lipschitz $k$-graphs $\mathcal{G}_{i} \subset \mathbb{R}^{N}$, such that

$$
\mathscr{H}^{k}\left(E \backslash \bigcup_{i=1}^{\infty} \mathcal{G}_{i}\right)=0 .
$$

In what follows, a particular case of [21, Theorem 3.2.22] is also used:

Theorem 2.4. Let $\Omega \subset \mathbb{R}^{3}$ be open, bounded and connected, $\mathcal{Z} \subset \mathbb{R}^{3}$ be a 1-rectifiable set and $\mathbf{f}: \Omega \rightarrow \mathcal{Z}$ a Lipschitz function. Define the 1-dimensional Jacobian of $\mathbf{f}$ by:

$$
J_{1} \mathbf{f}:=\sqrt{\sum_{i, j}(\nabla \mathbf{f})_{i j}^{2}} .
$$

Then:

- for $\mathscr{L}^{3}$ almost every $\mathbf{x} \in \Omega$, either $J_{1} \mathbf{f}(\mathbf{x})=0$, or the image of $\nabla \mathbf{f}(x)$ is a 1-dimensional vector space, i.e., rank $\nabla \mathbf{f}(\mathbf{x}) \leq 1, \mathscr{L}^{3}$-almost everywhere in $\Omega$;

- for $\mathscr{H}^{1}$ almost all $\boldsymbol{\xi} \in \mathcal{Z}, \mathbf{f}^{-1}(\boldsymbol{\xi})$ is 2-rectifiable;

- for every integrable $g: \Omega \rightarrow[-\infty, \infty]$

$$
\int_{\Omega} g(\mathbf{x}) J_{1} \mathbf{f}(\mathbf{x}) \mathrm{d} \mathbf{x}=\int_{\mathcal{Z}} \int_{\mathbf{f}^{-1}(\boldsymbol{\xi}) \cap \Omega} g(\mathbf{s}) \mathrm{d} \mathscr{H}^{2}(\mathbf{s}) \mathrm{d} \mathscr{H}^{1}(\boldsymbol{\xi})
$$

\section{The moving mask assumption}

The aim of this section is to give a precise definition of the moving mask assumption (see Definition 3.1 below), and to frame it in the context of dynamics for nonlinear continuum mechanics. This is done by recalling first the simplified model derived in [15] to describe the evolution of martensitic transformation in the context of nonlinear continuum mechanics. In this framework, we introduce some hypotheses approximating experimental observation. These hypotheses are made precise in Definition [3.1. We remark that the model in [15] is used here just to frame the moving mask assumption, and that the rest of the paper relies on Definition 3.1 only, which could be hence taken by the reader as a standalone assumption. 
In 15] we introduced a continuum model for the evolution of martensitic transformations. After passing to the limit in which the elastic constants tend to infinity and the interface energy density tends to zero, we deduced that the deformation gradients and the temperature field generate in the limit a Young measure $\nu_{\mathbf{x}, t}$ (see as a reference [29, 30]) and a function $\theta$ satisfying in a suitable sense

$$
\begin{aligned}
\rho_{0} \theta_{t}-d \Delta \theta & =-\theta_{T} \frac{\partial}{\partial t} \int_{\mathbb{R}^{3 \times 3}} \eta_{1}(\mathrm{~A}) \mathrm{d} \nu_{\mathbf{x}, t}(\mathrm{~A}), & & \text { a.e. in } \Omega \times(0, T), \\
\operatorname{supp} \nu_{\mathbf{x}, t} & \subset S O(3) \cup K, & & \text { a.e. in } \Omega \times(0, T),
\end{aligned}
$$

complemented with some initial and boundary conditions. Here, $\rho_{0}$ is the density of the body, $d$ is a diffusivity coefficient which is supposed to be constant, and $\eta_{1}$ is a smooth function such that

$$
\eta_{1}(\mathrm{~F})=0, \quad \text { for all } \mathrm{F} \in S O(3), \quad \eta_{1}(\mathrm{~F})=-\frac{\alpha}{\theta_{T}}, \quad \text { for all } \mathrm{F} \in K,
$$

for some constant $\alpha>0$ representing the latent heat of the transformation. This system of equations is underdetermined, and should therefore be closed with some constitutive relation between $\int_{\mathbb{R}^{3 \times 3}} \eta_{1}(\mathrm{~A}) \mathrm{d} \nu_{\mathbf{x}, t}(\mathrm{~A})$, and $\theta$ and $\nu_{\mathbf{x}, t}$. Nonetheless, we aim to characterise solutions independently of the constitutive relation. In order to do this we introduce some hypotheses on the solutions, that are based on experimental observation and that together we call the moving mask approximation, defined precisely in Definition 3.1 below, using the following ingredients:

- the phases are separated, that is there exist open sets $\Omega_{A}(t), \Omega_{M}(t) \subset \Omega$ such that

$$
\Omega_{A}(t) \cap \Omega_{M}(t)=\varnothing, \quad \mathscr{L}^{3}\left(\Omega \backslash\left(\Omega_{A}(t) \cup \Omega_{M}(t)\right)\right)=0, \quad \text { a.e. } t \in(0, T),
$$

and

$$
\begin{array}{r}
\nu_{\mathbf{x}, t}(S O(3))=1, \quad \text { a.e. } \mathbf{x} \in \Omega_{A}(t), \text { a.e. } t \in(0, T), \\
\nu_{\mathbf{x}, t}(K)=1, \quad \text { a.e. } \mathbf{x} \in \Omega_{M}(t), \text { a.e. } t \in(0, T) .
\end{array}
$$

The domain can hence be divided for almost every $t \in(0, T)$ into two regions, the region with martensite $\Omega_{M}(t)$, and the region with austenite $\Omega_{A}(t)$. Thus,

$$
\int_{\mathbb{R}^{3 \times 3}} \eta_{1}(\mathrm{~A}) \mathrm{d} \nu_{\mathbf{x}, t}(\mathrm{~A})=-\frac{\alpha}{\theta_{T}} \chi_{\Omega_{M}}(\mathbf{x}, t)
$$

where $\chi_{\Omega_{M}}(\mathbf{x}, t)$ is the characteristic function of $\Omega_{M}(t)$. The austenite-martensite phase boundary is sharp in this case. In terms of macroscopic deformation gradients this reads

$$
\begin{aligned}
& \nabla \mathbf{y}(\mathbf{x}, t) \in K^{q c}, \quad \text { a.e. in } \Omega_{M}(t), \text { a.e. } t \in(0, T), \\
& \nabla \mathbf{y}(\mathbf{x}, t) \in S O(3), \quad \text { a.e. in } \Omega_{A}(t), \text { a.e. } t \in(0, T),
\end{aligned}
$$

- during the phase transition, the macroscopic deformation gradient remains equal to a constant rotation in the austenite region. This is the case, for example, when the austenite region is connected;

- the phase interface moves continuously. More precisely, for almost every point $\mathbf{x}$ in the domain, there exists a time when $\mathbf{x}$ is contained in the phase interface (see also [15, Remark 5.2]); 
- the microstructures do not change after the transformation has happened. This assumption makes particular sense in the context of materials satisfying the cofactor conditions, where austenite and finely twinned martensite can be exactly compatible across interfaces, and even more in $\mathrm{Zn}_{45} \mathrm{Au}_{30} \mathrm{Cu}_{25}$ where the phase transition has very low thermal hysteresis and thermal expansion is hence negligible.

As remarked in the introduction, this construction reflects the idea of a moving mask that uncovers a martensitic microstructure, as can be seen in the video of [31]. Mathematically we can define the moving mask approximation as follows:

Definition 3.1. We say that $\nabla \mathbf{y} \in L^{\infty}\left(\Omega ; \mathbb{R}^{3 \times 3}\right)$ satisfies the moving mask approximation if

- for each $t \in[0, T]$ there exist $\Omega_{M}(t), \Omega_{A}(t) \subset \Omega$ disjoint and open, such that

$$
\mathscr{L}^{3}\left(\Omega \backslash\left(\Omega_{A}(t) \cup \Omega_{M}(t)\right)\right)=0 ;
$$

- either

$$
\Omega_{A}\left(t_{2}\right) \subset \Omega_{A}\left(t_{1}\right), \quad \text { for all } 0 \leq t_{1} \leq t_{2} \leq T,
$$

or

$$
\Omega_{A}\left(t_{1}\right) \subset \Omega_{A}\left(t_{2}\right), \quad \text { for all } 0 \leq t_{1} \leq t_{2} \leq T
$$

- for a.e. $\mathbf{x} \in \Omega$ there exists $t=t(\mathbf{x}) \in[0, T]$ such that $\mathbf{x} \in \bar{\Omega}_{A}(t) \cap \bar{\Omega}_{M}(t)$;

- there exists $\mathrm{Q} \in S O(3)$ such that for every $t \in[0, T]$ the map $\mathbf{y}_{M}(\cdot, t)$ satisfying

$$
\nabla \mathbf{y}_{M}(\mathbf{x}, t)= \begin{cases}\nabla \mathbf{y}(\mathbf{x}), & \text { a.e. in } \Omega_{M}(t) \\ \mathbf{Q}, & \text { a.e. in } \Omega_{A}(t)\end{cases}
$$

is in $W^{1, \infty}\left(\Omega ; \mathbb{R}^{3}\right)$.

Remark 3.1. We note that, in the case $\Omega_{M}(s) \subset \Omega_{M}(t)$ for each $s, t \in[0, T]$ with $s<t$, we have

$$
\bigcap_{t \in[0, T]} \Omega_{A}(t)=\varnothing, \quad \bigcup_{t \in[0, T]} \Omega_{M}(t)=\Omega .
$$

Remark 3.2. If we assume (3.18), then the formula for differentiation of integrals on time dependent domains implies that

$$
\begin{array}{r}
\left\langle\frac{\partial}{\partial t} \int_{\mathbb{R}^{3 \times 3}} \eta_{1}(\mathrm{~A}) \mathrm{d} \nu_{\mathbf{x}, t}(\mathrm{~A}), \psi\right\rangle=\left\langle\dot{\chi}_{\Omega_{M}}(\nabla \mathbf{y}), \psi\right\rangle=\frac{d}{d t} \int_{\Omega_{M}} \psi \mathrm{d} \mathbf{x} \\
=\int_{\Gamma(t)}(\mathbf{v} \cdot \mathbf{n}) \psi \mathrm{d} \mathscr{H}^{2}, \quad \forall \psi \in C_{0}^{\infty}(\Omega),
\end{array}
$$

provided $\Omega_{A}(t), \Omega_{M}(t)$ and $\mathbf{v} \cdot \mathbf{n}$ are smooth enough (see e.g., [22]). Here $\Gamma(t):=\Omega \backslash\left(\Omega_{A} \cup \Omega_{M}\right)(t)$ is a surface separating $\Omega_{A}(t)$ from $\Omega_{M}(t)$, $\mathbf{n}$ denotes the outer normal to $\Omega_{M}$ and $\mathbf{v}(\mathbf{s})$ is the velocity of the interface at the point $\mathbf{s} \in \Gamma(t)$ at time $t$. By $\langle\cdot, \cdot\rangle$ we denoted the duality pairing between a distribution and a test function. A version of (3.19) in the case of some solutions to (3.16) -(3.17) satisfying the moving mask assumptions is given by Corollary 4.4 below. 


\section{Generalized Hadamard conditions}

In this section we restrict our attention to deformation gradients $\nabla \mathbf{y}$ that satisfy the moving mask approximation as stated in Definition 3.1. In order to say something more about solutions under these assumptions, we prove below a variant of the Hadamard jump condition reflecting this hypothesis. In what follows, we restrict, without loss of generality, to the case $\Omega_{M}(s) \subset \Omega_{M}(t)$ for every $s<t$. As before, below $\Omega \subset \mathbb{R}^{3}$ is an open bounded connected set with Lipschitz boundary. For simplicity, rather than working with the deformation map $\mathbf{y}$, in this section we mostly work with the displacement $\operatorname{map} \mathbf{z}:=\mathbf{y}-\mathbf{Q x}$, where $\mathbf{Q}$ is as in Definition 3.1.

We start by proving the result when the phase interfaces are planar. This situation describes, for example, the propagation of a simple martensitic laminate in the austenite phase.

Proposition 4.1. Let $\Gamma(t)$ be a family of parallel planes perpendicular to $\mathbf{n} \in \mathbb{S}^{2}$,

$$
\Gamma(t):=\left\{\mathbf{x} \in \mathbb{R}^{3}: \mathbf{x} \cdot \mathbf{n}=h(t)\right\},
$$

for some non-decreasing function $h \in C([0, T])$ satisfying

$$
h(0)=\inf _{\mathbf{x} \in \Omega} \mathbf{x} \cdot \mathbf{n}, \quad h(T)=\sup _{\mathbf{x} \in \Omega} \mathbf{x} \cdot \mathbf{n} .
$$

For $t \in[0, T]$ define

$$
\Omega_{M}(t):=\Omega \cap\{\mathbf{x} \cdot \mathbf{n}<h(t)\}, \quad \Omega_{A}(t):=\Omega \cap\{\mathbf{x} \cdot \mathbf{n}>h(t)\} .
$$

Let $\mathbf{z} \in W^{1, \infty}\left(\Omega ; \mathbb{R}^{3}\right)$ be such that $\mathbf{Z}=\mathbf{Z}(\mathbf{x}, t)$ satisfying

$$
\nabla \mathbf{Z}(\mathbf{x}, t)= \begin{cases}\nabla \mathbf{z}(\mathbf{x}), & \text { a.e. } \text { in } \Omega_{M}(t) \\ 0, & \text { a.e. in } \Omega_{A}(t)\end{cases}
$$

is in $W^{1, \infty}\left(\Omega ; \mathbb{R}^{3}\right)$ for a.e. $t \in(0, T)$. Then,

1. there exists $\mathbf{a} \in L^{\infty}\left(\Omega ; \mathbb{R}^{3}\right)$ such that

$$
\nabla \mathbf{z}(\mathbf{x})=\mathbf{a}(\mathbf{x}) \otimes \mathbf{n}, \quad \text { a.e. } \mathbf{x} \in \Omega .
$$

2. if $\Omega \cap \Gamma(t)$ is connected for every $t \in(0, T)$ then $\mathbf{z}=\mathbf{f}(\mathbf{x} \cdot \mathbf{n})$ for some $\mathbf{f} \in W^{1, \infty}\left((0, T) ; \mathbb{R}^{3}\right)$.

Proof. By rotating the system of coordinates we can assume without loss of generality that $\mathbf{n}=\mathbf{e}_{3}$. Let us consider the set $\mathcal{B}_{1} \subset \Omega$ of points where $\mathbf{z}$ is differentiable, and the set $\mathcal{B}_{2}$ of points $\mathbf{x} \in \Omega$ such that there exists $t^{*} \in(0, T)$ for which $\mathbf{x} \in \Gamma\left(t^{*}\right)$ and $\mathbf{Z}\left(\cdot, t^{*}\right) \in W^{1, \infty}\left(\Omega ; \mathbb{R}^{3}\right)$. By continuity of $h$ we have that $\mathscr{L}^{3}\left(\Omega \backslash\left(\mathcal{B}_{1} \cap \mathcal{B}_{2}\right)\right)=0$. Let us thus consider a generic point $\hat{\mathbf{x}} \in \mathcal{B}_{1} \cap \mathcal{B}_{2}$, and notice that, since $\Omega$ is open, there exists $r>0$ such that the ball $B(\hat{\mathbf{x}}, r) \subset \Omega$. By (4.20), $\mathbf{Z}\left(\cdot, t^{*}\right)$ must be constant in each connected component of $\Omega_{A}(t)$. In particular, as $\mathbf{Z}\left(\cdot, t^{*}\right) \in W^{1, \infty}\left(\Omega, \mathbb{R}^{3}\right)$ is continuous, it must be a constant on $\Gamma\left(t^{*}\right) \cap B(\hat{\mathbf{x}}, r)$. At the same time, continuity of $\mathbf{z}$ and $\mathbf{Z}\left(\cdot, t^{*}\right)$ implies also $\mathbf{z}(\mathbf{x})=\mathbf{Z}\left(\mathbf{x}, t^{*}\right)$ for every $\mathbf{x} \in \Gamma\left(t^{*}\right) \cap B(\hat{\mathbf{x}}, r)$. Therefore, the function $\mathbf{z}(\mathbf{x})$ must be constant on $\Gamma\left(t^{*}\right) \cap B(\hat{\mathbf{x}}, r)$. This implies,

$$
\frac{\partial \mathbf{z}}{\partial x_{i}}(\hat{\mathbf{x}})=0, \quad i=1,2
$$


The arbitrariness of $\hat{\mathbf{x}} \in \mathcal{B}_{1} \cap \mathcal{B}_{2}$ yields the first statement. On the other hand, if $\Omega \cap \Gamma(t)$ is connected for a.e. $t \in(0, T)$, then $\mathbf{z}(\mathbf{x})$ is constant on $\Gamma\left(t^{*}\right) \cap \Omega$ for a.e. $t \in(0, T)$ and hence $\mathbf{z}=\mathbf{z}\left(x_{3}\right)$. This concludes the proof.

Remark 4.1. We could replace the hypothesis concerning the connectedness of $\Omega_{A}(t)$ by assuming that $\mathbf{Z}(\mathbf{x}, t)$ is equal to a constant $\mathbf{c}(t) \in \mathbb{R}^{3}$ in $\Omega_{A}(t)$ for a.e. $t \in[0, T]$. Both these assumptions are automatically satisfied if $\Omega$ is convex.

Remark 4.2. In the case $\mathbf{z}=\mathbf{f}(\mathbf{x} \cdot \mathbf{n})$, and $\Gamma(t)$ is a single plane, the phase interfaces must coincide with the level sets of $\mathbf{f}$. Therefore, given an experimentally measured martensitic macroscopic deformation gradient, under the assumption that it satisfies the moving mask approximation, and is of the form $1+\mathbf{a}(\mathbf{x} \cdot \mathbf{n}) \otimes \mathbf{n}$, for some $\mathbf{a} \in \mathbb{R}^{3}, \mathbf{n} \in \mathbb{S}^{2}$, we can reconstruct the position of austenite-martensite phase interfaces, by taking the level sets of $\mathbf{f}(\mathbf{x} \cdot \mathbf{n})=\int_{0}^{\mathbf{x} \cdot n} \mathbf{a}(s) \mathrm{d} s$. Furthermore, in the case $\mathbf{z}=\mathbf{f}(\mathbf{x} \cdot \mathbf{n})$, and $\Gamma(t)$ is a single plane, the discontinuities in the macroscopic deformation gradient can occur only across the planes $\mathbf{x} \cdot \mathbf{n}=$ constant. This is, for example, the case for type II twins satisfying the cofactor conditions, for which we refer the reader to Proposition 6.2 .

Proposition 4.1 can be partially generalized to the case where $\Gamma(t)$ is a family of curved interfaces. As a first step, we need to introduce the concept of moving interfaces for our problem, generalizing the previous requirements on planar such interfaces.

Definition 4.1. We say that $\Gamma(t) \subset \Omega$ is a family of moving interfaces in $\Omega$ if:

(i) there exist two families of open disjoint sets $\Omega_{M}(t), \Omega_{A}(t) \subset \Omega$ and a bounded open interval $I_{T}:=[0, T]$ such that for every $t$ in $I_{T}$,

$$
\Omega=\Omega_{M}(t) \cup \Omega_{A}(t) \cup \Gamma(t) \quad \text { and } \quad \Gamma(t) \cap \Omega_{M}(t)=\Gamma(t) \cap \Omega_{A}(t)=\varnothing .
$$

Furthermore, $\Omega_{M}(t)$ is non-decreasing in $t$, i.e.,

$$
\Omega_{M}(t) \subset \Omega_{M}(s), \quad \Omega_{A}(s) \subset \Omega_{A}(t), \quad \forall t<s \in I_{T}
$$

(ii) the set

$$
\mathcal{B}:=\left\{\begin{array}{l|c}
\mathbf{x} \in \Omega \mid \Gamma\left(t^{*}\right), t^{*} \in I_{T} \text { and there exist } \mathcal{U}_{\mathbf{x}} \subset \Omega \text { open } \\
\text { and connected, } \mathbf{x} \in \mathcal{U}_{\mathbf{x}} \text {, and a Lipschitz 2-graph } \\
\mathcal{G}_{\mathbf{x}} \text { differentiable at } \mathbf{x} \text { such that } \\
\mathcal{G}_{\mathbf{x}} \cap \mathcal{U}_{\mathbf{x}} \subset \Gamma\left(t^{*}\right) \cap \mathcal{U}_{\mathbf{x}} \subset \bar{\Omega}_{M}\left(t^{*}\right) \cap \bar{\Omega}_{A}\left(t^{*}\right) \cap \mathcal{U}_{\mathbf{x}}
\end{array}\right\}
$$

is measurable and $\mathscr{L}^{3}(\Omega \backslash \mathcal{B})=0$.

Points in $\mathcal{B}$ are called regular points for $\Gamma(t)$.

At this point we can also introduce the concept of a regular moving mask approximation:

Definition 4.2. We say that $\mathbf{y} \in W^{1, \infty}\left(\Omega ; \mathbb{R}^{3}\right)$ satisfies a regular moving mask approximation if it satisfies the moving mask approximation and

$$
\Gamma(t)=\Omega \backslash\left(\Omega_{A}(t) \cup \Omega_{M}(t)\right)
$$

is a family of moving interfaces in $\Omega$, where $\Omega_{A}, \Omega_{M}$ are as in Definition 3.1 . 


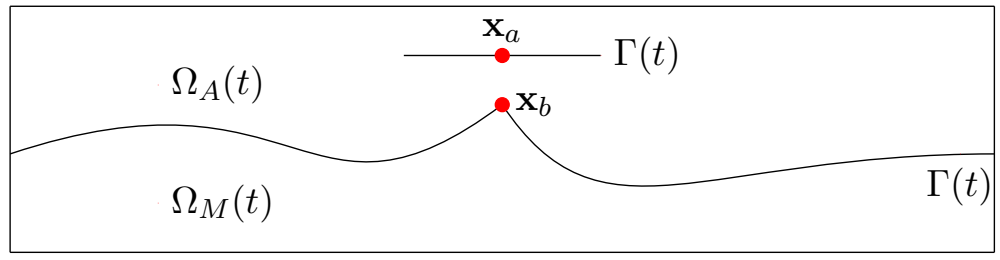

Figure 1: Points which are not regular: $\mathbf{x}_{a}$ is in a smooth $k$-graph contained in $\Gamma(t)$, but is not separating $\Omega_{A}$ from $\Omega_{M}$. $\Gamma(t)$ does not coincide with a Lipschitz function differentiable at $\mathbf{x}_{b}$.

Remark 4.3. The requirement $\Gamma\left(t^{*}\right) \cap \mathcal{U} \subset \bar{\Omega}_{A}\left(t^{*}\right) \cap \bar{\Omega}_{M}\left(t^{*}\right) \cap \mathcal{U}$ in Definition 4.1(iii) is mainly to guarantee that the set where an interface is cutting either $\Omega_{A}$ or $\Omega_{M}$ and not separating one from the other is small (see e.g., the point $\mathbf{x}_{a}$ in Figure 1). In this way, families of moving interfaces satisfying the separation condition may also describe further nucleations in the interior of $\Omega_{A}$ during the phase transition.

Below, we say that a curve $\mathbf{c}:\left[t_{0}, t_{1}\right] \rightarrow \mathbb{R}^{3}$, for some $t_{0}, t_{1} \in \mathbb{R}$, is simple if $\mathbf{c}(s) \neq \mathbf{c}(t)$ for each $s, t \in\left[t_{0}, t_{1}\right]$. The following theorem generalizes Proposition 4.1 to curved interfaces:

Theorem 4.1. Let $\Gamma(t)$ be a family of moving interfaces in $\Omega$. Assume $\mathbf{z} \in W^{1, \infty}\left(\Omega ; \mathbb{R}^{3}\right)$ is such that the function $\mathbf{Z}=\mathbf{Z}(\mathbf{x}, t)$ satisfying

$$
\begin{cases}\nabla \mathbf{z}(\mathbf{x}), & \text { a.e. in } \Omega_{M}(t) \\ 0, & \text { a.e. in } \Omega_{A}(t)\end{cases}
$$

with $\Omega_{A}(t), \Omega_{M}(t)$ as in Definition 4.1, is in $W^{1, \infty}\left(\Omega ; \mathbb{R}^{3}\right)$ for every $t \in I_{T}$. Then, there exist $\mathbf{a} \in$ $L^{\infty}\left(\Omega ; \mathbb{R}^{3}\right), \mathbf{n} \in L^{\infty}\left(\Omega ; \mathbb{S}^{2}\right)$ such that

$$
\nabla \mathbf{z}(\mathbf{x})=\mathbf{a}(\mathbf{x}) \otimes \mathbf{n}(\mathbf{x}), \quad \text { a.e. } \mathbf{x} \in \Omega .
$$

Conversely, let $\mathbf{z} \in W^{1, \infty}\left(\Omega ; \mathbb{R}^{3}\right)$ be such that (4.22) is satisfied and $\mathbf{z}(\Omega)$ is contained in the image of an absolutely continuous simple curve $\mathbf{c}: I_{T} \rightarrow \mathbb{R}^{3}$ of finite length. Then, if $|\mathbf{a}|>0$ a.e. in $\Omega$, there exists a family of moving interfaces in $\Omega$, and a $\mathbf{Z}=\mathbf{Z}(\mathbf{x}, t)$ in $W^{1, \infty}\left(\Omega ; \mathbb{R}^{3}\right)$ satisfying (4.21) for every $t \in I_{T}$. Furthermore, $\mathscr{L}^{3}\left(\Omega \backslash\left(\Omega_{A}(t) \cup \Omega_{M}(t)\right)\right)=0$ for every $t \in I_{T}$.

Remark 4.4. The assumptions on the image of $\mathbf{z}$ in Theorem 4.1 are motivated by the following observation: if $\mathbf{z} \in C^{1}\left(\Omega ; \mathbb{R}^{3}\right)$, and $\nabla \mathbf{z}$ is rank-one everywhere in $\Omega$, then the constant rank theorem implies that, around every $\mathbf{x} \in \Omega$, the image of $\mathbf{z}$ is a simple absolutely continuous curve. However, the set $\mathbf{z}(\Omega)$ can a priori show branching and other complex structures even in the regular case (e.g., if $\Omega$ is non-convex). For the sake of clarity of the proof, in this paper we restrict ourselves to the easier case where $\mathbf{z}(\Omega)$ is a simple absolutely continuous curve. Nonetheless, a statement similar to the second implication in Theorem 4.1 can be proved for maps $\mathbf{z}: \Omega \rightarrow \mathbb{R}^{3}$ whose image satisfies

- $\mathbf{z}(\Omega)$ is 1 -rectifiable;

- for $\mathscr{H}^{1}$-a.e. $\boldsymbol{\xi} \in \mathbf{z}(\Omega)$ there exist an open ball $\mathcal{D}_{\boldsymbol{\xi}} \subset \mathbb{R}^{3}$ such that $\mathcal{D}_{\boldsymbol{\xi}} \cap \mathbf{z}(\Omega)$ is a simple curve of finite length which is absolutely continuous. 
Remark 4.5. Assume that the moving mask assumption holds, and that we can reconstruct $\mathbf{z}$ from experimental observations. Then, provided the image of $\mathbf{z}$ satisfies the stated assumptions, the second part of Theorem 4.1 gives a useful tool to reconstruct phase interfaces during the phase transformation.

For the proof of Theorem 4.1 we need the following Lemma:

Lemma 4.1. Let $\mathrm{f} \in L^{\infty}\left(\Omega ; \mathbb{R}^{3 \times 3}\right)$ be such that $\mathrm{f}(\mathbf{x})=(\mathbf{b} \otimes \mathbf{m})(\mathbf{x})$ for a.e. $\mathbf{x} \in \Omega$. Then, there exist $\mathbf{a}, \mathbf{n} \in L^{\infty}\left(\Omega ; \mathbb{R}^{3}\right)$ such that $\mathbf{f}(\mathbf{x})=\mathbf{a}(\mathbf{x}) \otimes \mathbf{n}(\mathbf{x})$ and $|\mathbf{n}(\mathbf{x})|=1$ for a.e. $\mathbf{x} \in \Omega$.

Proof. This is just a matter of measurability of $\mathbf{a}, \mathbf{n}$. As $\mathbf{f}$ is measurable, so is $f^{T} \mathbf{f}=\left(|\mathbf{b}|^{2} \mathbf{m} \otimes \mathbf{m}\right)$, so is its trace $\operatorname{tr}\left(\mathbf{f}^{T} \mathbf{f}\right)=|\mathbf{b}|^{2}|\mathbf{m}|^{2}$ and so is the function

$$
g:= \begin{cases}|\mathbf{b}|^{-2}|\mathbf{m}|^{-2} \mathbf{f}^{T} \mathbf{f}, & \text { if }|\mathbf{b}|^{2}|\mathbf{m}|^{2} \neq 0 \\ 0, & \text { otherwise. }\end{cases}
$$

Therefore, we define $\Omega_{1}:=\left\{\mathbf{x} \in \Omega: g_{11}(\mathbf{x}) \neq 0\right\}$ and

$$
\mathbf{n}(\mathbf{x})=\left(\left(g_{11}(\mathbf{x})\right)^{\frac{1}{2}}, g_{12}(\mathbf{x})\left(g_{11}(\mathbf{x})\right)^{-\frac{1}{2}}, g_{13}(\mathbf{x})\left(g_{11}(\mathbf{x})\right)^{-\frac{1}{2}}\right)^{T},
$$

for almost every $\mathbf{x} \in \Omega_{1}$. This is actually possible because $g_{i i} \geq 0$ a.e. in $\Omega$, for $i=1,2,3$. Define also

$$
\Omega_{2}:=\left\{\mathbf{x} \in \Omega \backslash \Omega_{1}: g_{22} \neq 0\right\}, \quad \Omega_{3}:=\left\{\mathbf{x} \in \Omega \backslash\left(\Omega_{1} \cup \Omega_{2}\right): g_{33} \neq 0\right\},
$$

and define $n$ in $\Omega_{2}, \Omega_{3}$ respectively by

$$
\begin{aligned}
& \mathbf{n}=\left(g_{21}\left(g_{22}\right)^{-\frac{1}{2}},\left(g_{22}\right)^{\frac{1}{2}}, g_{32}\left(g_{22}\right)^{-\frac{1}{2}}\right)^{T}, \\
& \mathbf{n}=\left(g_{31}\left(g_{33}\right)^{-\frac{1}{2}}, g_{32}\left(g_{33}\right)^{-\frac{1}{2}},\left(g_{33}\right)^{\frac{1}{2}}\right)^{T} .
\end{aligned}
$$

Therefore, choosing $\mathbf{n}$ arbitrarily and such that $|\mathbf{n}|=1$ in the set where $\mathrm{g}=0$, we have constructed $\mathbf{n} \in L^{\infty}\left(\Omega ; \mathbb{R}^{3}\right)$ as desired. Defining $\mathbf{a}:=\mathbf{f} \mathbf{n}$, we thus conclude the proof.

Proof of Theorem 4.1. We first prove (4.22).

Let $N_{\mathbf{z}}$ be the set where $\mathbf{z}$ is not differentiable and remark that, by the hypotheses, $N:=N_{\mathbf{z}} \cup(\Omega \backslash \mathcal{B})$ is an $\mathscr{L}^{3}$-negligible set. Let $\mathbf{x}_{0} \in \Omega \backslash N$ and take $\mathcal{U}_{\mathbf{x}_{0}}$ to be a neighbourhood of $\mathbf{x}_{0}$ as in Definition 4.1 (ii). By taking a smaller connected neighbourhood of $\mathbf{x}_{0}$, which we still denote by $\mathcal{U}_{\mathbf{x}_{0}}$, we can assume that $\mathcal{G}_{\mathbf{x}_{0}} \cap \mathcal{U}_{\mathbf{x}_{0}}$ is connected. We first claim that $\mathbf{z}$ is constant on $\mathcal{G}_{\mathbf{x}_{0}} \cap \mathcal{U}_{\mathbf{x}_{0}}$. Indeed, as $\nabla \mathbf{Z}\left(\mathbf{x}, t^{*}\right)=0$ a.e. in $\Omega_{A}\left(t^{*}\right)$, the continuity of $\mathbf{Z}\left(\mathbf{x}, t^{*}\right)$ implies that $\mathbf{Z}\left(\mathbf{x}, t^{*}\right)$ must be constant on every connected component of $\bar{\Omega}_{A}\left(t^{*}\right)$. Since Definition 4.1 (iii) implies $\mathcal{G}_{\mathbf{x}_{0}} \cap \mathcal{U}_{\mathbf{x}_{0}} \subset \bar{\Omega}_{A}\left(t^{*}\right)$, we must have $\mathbf{Z}\left(\cdot, t^{*}\right)=\hat{\mathbf{c}}$ for some $\hat{\mathbf{c}} \in \mathbb{R}^{3}$ on $\mathcal{G}_{\mathbf{x}_{0}} \cap \mathcal{U}_{\mathbf{x}_{0}}$. On the other hand, continuity of $\mathbf{z}, \mathbf{Z}\left(\cdot, t^{*}\right)$ together with (4.21) imply that on every connected component of $\bar{\Omega}_{M}\left(t^{*}\right) \mathbf{z}=\mathbf{Z}\left(\cdot, t^{*}\right)+\overline{\mathbf{c}}$ for some $\overline{\mathbf{c}} \in \mathbb{R}^{3}$ depending on the connected component. Therefore, as by Definition 4.1 (ii) $\mathcal{G}_{\mathbf{x}_{0}} \cap \mathcal{U}_{\mathbf{x}_{0}} \subset \bar{\Omega}_{M}\left(t^{*}\right)$, the fact that $\mathbf{Z}\left(\cdot, t^{*}\right)$ is constant on $\mathcal{G}_{\mathbf{x}_{0}} \cap \mathcal{U}_{\mathbf{x}_{0}}$ implies that so must be $\mathbf{z}$.

Now, as $\mathcal{G}_{\mathbf{x}_{0}}$ is a Lipschitz 2-graph, we can find a Lipschitz change of coordinates $\boldsymbol{\psi}: \mathcal{U}_{\mathbf{x}_{0}} \rightarrow V$ such that

$$
\boldsymbol{\psi}\left(\mathcal{G}_{\mathbf{x}_{0}} \cap \mathcal{U}_{\mathbf{x}_{0}}\right)=\left\{\mathbf{x} \in \mathbb{R}^{3}: \mathbf{x} \cdot \mathbf{n}\left(\mathbf{x}_{0}\right)=c_{\Gamma}\right\} \cap V
$$


for some open connected $V \subset \mathbb{R}^{3}, c_{\Gamma} \in \mathbb{R}$ and where $\mathbf{n}\left(\mathbf{x}_{0}\right)$ is the normal vector to $\mathcal{G}_{\mathbf{x}_{0}}$ at $\mathbf{x}_{0}$ pointing outwards from $\Omega_{M}\left(t^{*}\right)$. Let us denote $\boldsymbol{\psi}(\mathbf{x})=\overline{\mathbf{x}}$ for every $\mathbf{x} \in \mathcal{U}_{\mathbf{x}_{0}}$. We define $\overline{\mathbf{z}}$ as $\overline{\mathbf{z}}(\overline{\mathbf{x}})=\mathbf{z}\left(\boldsymbol{\psi}^{-1}(\overline{\mathbf{x}})\right)$, and assuming without loss of generality that $\mathbf{n}(\mathbf{x})=\mathbf{e}_{3}$, we get that

$$
\overline{\mathbf{z}}\left(\overline{\mathbf{x}}_{0}\right)=\overline{\mathbf{z}}\left(\overline{\mathbf{x}}_{0}+s \mathbf{e}_{i}\right)=\hat{\mathbf{c}}, \quad i=1,2,
$$

for each $s$ such that $\overline{\mathbf{x}}_{0}+s \mathbf{e}_{i} \in V$. This is due to the fact that $\mathbf{z}(\mathbf{x})=\hat{\mathbf{c}}$ for every $\mathbf{x} \in \mathcal{U}_{\mathbf{x}_{0}} \cap \mathcal{G}_{\mathbf{x}_{0}}$. Therefore,

$$
\frac{\partial \overline{\mathbf{z}}}{\partial \bar{x}_{i}}(\overline{\mathbf{x}})=0, \quad i=1,2 .
$$

On the other hand, as $\mathbf{x}_{0} \in \Omega \backslash N$, we have

$$
\nabla_{\mathbf{x}} \mathbf{z}\left(\mathbf{x}_{0}\right)=\nabla_{\overline{\mathbf{x}}} \overline{\mathbf{z}}\left(\boldsymbol{\psi}\left(\mathbf{x}_{0}\right)\right) \nabla_{\mathbf{x}} \boldsymbol{\psi}\left(\mathbf{x}_{0}\right) .
$$

Since $\mathbf{x}_{0}$ is a regular point, $\boldsymbol{\psi}$ can be chosen to be differentiable in $\mathbf{x}_{0}$, and therefore $\overline{\mathbf{x}}_{0}$ is a point of differentiability for $\overline{\mathbf{z}}$. Therefore, there exists $\mathbf{a} \in \mathbb{R}^{3}$ such that

$$
\nabla_{\overline{\mathbf{x}}} \overline{\mathbf{z}}\left(\overline{\mathbf{x}}_{0}\right)=\mathbf{a} \otimes \mathbf{n}\left(\mathbf{x}_{0}\right)
$$

By putting together the last two identities and using the fact that $N$ is negligible we finally deduce (4.22). Measurability of $\mathbf{a}, \mathbf{n}$ follows from Lemma 4.1,

We now prove the second statement. We first remark that since $\mathbf{c}$ is absolutely continuous and of finite length, it belongs also to $W^{1,1}\left(I_{T} ; \mathbb{R}^{3}\right)$ and there exists $\mathbf{d} \in W^{1, \infty}\left(I_{T}^{*} ; \mathbb{R}^{3}\right)$ for some interval $I_{T}^{*} \subset \mathbb{R}$ such that $\mathbf{c}\left(I_{T}\right)=\mathbf{d}\left(I_{T}^{*}\right)$ (see e.g., [1] and references therein). Therefore $\mathbf{c}\left(I_{T}\right)$ is 1-rectifiable. By Theorem 2.4, $\Gamma(t):=\mathbf{z}^{-1}(\mathbf{c}(t)) \cap \Omega$ are 2-rectifiable surfaces for almost every $t$, and $\mathbf{z}$ is equal to a constant on them. Defining

$$
\begin{aligned}
\Omega_{M}(t) & :=\left\{\mathbf{x} \in \Omega: \exists s \in[0, t) \text { such that } \mathbf{x} \in \mathbf{z}^{-1}(\mathbf{c}(s))\right\}, \\
\Omega_{A}(t) & :=\left\{\mathbf{x} \in \Omega: \mathbf{x} \notin \mathbf{z}^{-1}(\mathbf{c}(s)), \forall s \in[0, t]\right\},
\end{aligned}
$$

it is easy to see that Definition 4.1(1i) is satisfied, provided we can show that $\Omega_{A}(t), \Omega_{M}(t)$ are open. To this end, let us fix $t^{*} \in I_{T}, \hat{\mathbf{x}} \in \Omega_{M}\left(t^{*}\right)$, and let us denote by $s_{\hat{\mathbf{x}}} \in\left[0, t^{*}\right)$ the point such that $\mathbf{z}(\hat{\mathbf{x}})=\mathbf{c}\left(s_{\hat{\mathbf{x}}}\right)$. As $\mathbf{z}$ is Lipschitz, we can define $R:=\frac{1}{2}\|\nabla \mathbf{z}\|_{L^{\infty}}^{-1}\left|\mathbf{c}\left(t^{*}\right)-\mathbf{z}(\hat{\mathbf{x}})\right|$, so that

$$
|\mathbf{z}(\mathbf{x})-\mathbf{z}(\hat{\mathbf{x}})| \leq\|\nabla \mathbf{z}\|_{L^{\infty}}|\mathbf{x}-\hat{\mathbf{x}}| \leq \frac{1}{2}\left|\mathbf{c}\left(t^{*}\right)-\mathbf{z}(\hat{\mathbf{x}})\right|=\frac{1}{2}\left|\mathbf{c}\left(t^{*}\right)-\mathbf{c}\left(s_{\hat{\mathbf{x}}}\right)\right|,
$$

for all $\mathbf{x} \in B_{R}(\hat{\mathbf{x}})$. Suppose now that in $B_{R}(\hat{\mathbf{x}})$ there exists a point $\mathbf{x}_{0}$ such that $\mathbf{z}\left(\mathbf{x}_{0}\right)=\mathbf{c}\left(t_{0}\right)$ for some $t_{0} \geq t$. Then the segment connecting $\mathbf{x}_{0}$ to $\hat{\mathbf{x}}$ is still contained in $B_{R}(\hat{\mathbf{x}})$, and its image through $\mathbf{z}$ must be a connected part of the image of $\mathbf{c}$. But as $\mathbf{c}$ is a simple curve, this implies that there exists $\mathbf{x}_{1} \in B_{R}(\hat{\mathbf{x}})$ such that $\mathbf{z}\left(\mathbf{x}_{1}\right)=\mathbf{c}\left(t^{*}\right)$, which is in contradiction with (4.24). Therefore, for every $t^{*} \in I_{T}, \hat{\mathbf{x}} \in \Omega_{M}\left(t^{*}\right)$ there exists an open ball centred at $\hat{\mathbf{x}}$ contained in $\Omega_{M}\left(t^{*}\right)$, and therefore $\Omega_{M}\left(t^{*}\right)$ is open. The same argument can be used to show that also $\Omega_{A}(t)$ is open for each $t$. Clearly, $\Gamma(t)$ is sequentially closed in $\Omega$ and $\Omega_{M}(t) \cup \Gamma(t), \Omega_{A}(t) \cup \Gamma(t)$ are closed in $\Omega$ as well. In this way we have also shown that $\mathbf{Z}(x, t)$ defined as

$$
\mathbf{Z}(x, t)= \begin{cases}\mathbf{z}(x), & \text { in } \Omega_{M}(t) \\ \mathbf{c}(t), & \text { in } \Omega_{A}(t)\end{cases}
$$


is in $W^{1, \infty}\left(\Omega ; \mathbb{R}^{3}\right)$ for every $t \in I_{T}$.

Now, since $\Gamma(t)$ is 2-rectifiable for almost every $t$, in order to show that

$$
\mathscr{L}^{3}\left(\Omega \backslash\left(\Omega_{A}(t) \cup \Omega_{M}(t)\right)\right)=0
$$

for every $t \in I_{T}$ it is sufficient to prove that

$$
\mathcal{C}:=\left\{\mathbf{x} \in \Omega: \mathbf{x} \in \Gamma(t), t \in I_{T}, \Gamma(t) \text { is not 2-rectifiable }\right\}
$$

has null $\mathscr{L}^{3}$ measure. By Theorem 2.4, $\mathcal{C}$ is the preimage through $\mathbf{z}$, which is continuous, of a set of measure zero, and is hence measurable. Now, we notice that by choosing $g$ to be the indicator function on $\mathcal{C}$ in the coarea formula (2.15), and identifying $\mathcal{Z}$ with the support of $\mathbf{c}$, we have

$$
0 \leq \int_{\Omega} g(\mathbf{x})|\mathbf{a}| \mathrm{d} \mathbf{x}=\int_{\mathcal{Z}} \int_{\mathbf{z}^{-1}(\boldsymbol{\xi})} g(\mathbf{s}) \mathrm{d} \mathscr{H}^{2}(\mathbf{s}) \mathrm{d} \mathscr{H}^{1}(\boldsymbol{\xi})=0
$$

as, by Theorem 2.4. this can just happen for a set of measure zero in $\mathcal{Z}$. This, together with the fact that $|\mathbf{a}|>0$ a.e., leads to $\mathscr{L}^{3}(\mathcal{C})=0$.

The rest of the proof is devoted to prove that Definition 4.1 (iii) is satisfied. To this aim, we first claim that for every point $\mathbf{x} \in \mathcal{D}$, with

$$
\mathcal{D}:=\{\mathbf{x} \in \Omega: \nabla \mathbf{z}(\mathbf{x}) \text { exists, and } \nabla \mathbf{z}(\mathbf{x}) \neq 0\}
$$

there exist a Lipschitz 2-graph $\mathcal{G}_{\mathbf{x}}$ which is differentiable at $\mathbf{x}$, an open neighbourhood $\mathcal{U}_{\mathbf{x}}$ and a $t^{*} \in I_{T}$ satisfying $\mathcal{G}_{\mathbf{x}} \cap \mathcal{U}_{\mathbf{x}} \subset \Gamma\left(t^{*}\right) \cap \mathcal{U}_{\mathbf{x}}$. In order to do that, we would need a generalised version of the constant rank theorem. However we were not able to find a version of it in the literature suitable to our application. We hence strongly exploit the structure of the image of $\mathbf{z}$ and a weak version of the implicit function theorem. Here and below, given a vector $\mathbf{v} \in \mathbb{R}^{3}$, we denote by $v_{i}$ its $i$-th component. Let us consider a generic $\hat{\mathbf{x}} \in \mathcal{D}$ and suppose, without loss of generality, that $a_{1}(\hat{\mathbf{x}}) \neq 0$ and that $\mathbf{n}(\hat{\mathbf{x}})=\mathbf{e}_{3}$. In this case, a version of the implicit function theorem as the one in [24, Thm. E] gives the existence of a connected neighbourhood $\mathcal{N}$ of $\left(\hat{x}_{1}, \hat{x}_{2}\right)$, and of a function $\psi: \mathcal{N} \rightarrow \mathbb{R}$, such that $\psi\left(\hat{x}_{1}, \hat{x}_{2}\right)=\hat{x}_{3}$, and $z_{1}\left(x_{1}, x_{2}, \psi\left(x_{1}, x_{2}\right)\right)=z_{1}(\hat{\mathbf{x}})$ for every $\left(x_{1}, x_{2}\right) \in \mathcal{N}$. Furthermore $\psi$ is differentiable in $\left(\hat{x}_{1}, \hat{x}_{2}\right)$ and hence continuous and Lipschitz in $\mathcal{N}$, and $\nabla \psi\left(\hat{x}_{1}, \hat{x}_{2}\right)=\mathbf{0}$.

Fixed $\varepsilon=\frac{\left|a_{1}(\hat{\mathbf{x}})\right|}{2}$, the fact that $\mathbf{z}$ is differentiable in $\hat{\mathbf{x}}$ implies the existence of $\delta>0$ such that

$$
z_{1}\left(\hat{\mathbf{x}}+\rho \mathbf{e}_{3}\right)-z_{1}(\hat{\mathbf{x}})=\rho a_{1}+r \rho, \quad \forall|\rho|<\delta,
$$

and where $|r|<\varepsilon$. Therefore,

$$
\begin{gathered}
z_{1}\left(\hat{\mathbf{x}}+\rho \mathbf{e}_{3}\right)>z_{1}(\hat{\mathbf{x}}), \quad \text { if } a_{1}(\hat{\mathbf{x}}) \delta>a_{1}(\hat{\mathbf{x}}) \rho>0, \\
z_{1}\left(\hat{\mathbf{x}}+\rho \mathbf{e}_{3}\right)<z_{1}(\hat{\mathbf{x}}), \quad \text { if }-a_{1}(\hat{\mathbf{x}}) \delta<a_{1}(\hat{\mathbf{x}}) \rho<0
\end{gathered}
$$

for all $|\rho|<\delta$. This implies the existence of $h>0$ and $\mathbf{c}\left(t^{*}+h\right), \mathbf{c}\left(t^{*}-h\right)$ in $\mathbf{z}(\Omega)$ such that

$$
c_{1}\left(t^{*}+h\right)>c_{1}\left(t^{*}\right)>c_{1}\left(t^{*}-h\right) .
$$


Here, $t^{*} \in I_{T}$ is such that $\mathbf{z}(\hat{\mathbf{x}})=\mathbf{c}\left(t^{*}\right)$. Furthermore, since $\mathbf{z}$ is Lipschitz, the dependence of $c(t(\mathbf{x})):=\mathbf{z}(\mathbf{x})$ is continuous. This together with (4.26) and the fact that $\mathbf{c}$ is simple, imply that the unique path connecting $\mathbf{c}\left(t^{*} \pm h\right)$ to $\mathbf{c}\left(t^{*}\right)$ must be such that $c_{1}\left(t^{*}+s\right)>c_{1}\left(t^{*}\right)>c_{1}\left(t^{*}-s\right)$ either for every $s \in(0, h)$ or for every $s \in(-h, 0)$. Suppose now the existence of $\left(x_{1}, x_{2}\right) \in \mathcal{N}$ such that $\mathbf{z}\left(x_{1}, x_{2}, \psi\left(x_{1}, x_{2}\right)\right) \neq \mathbf{c}\left(t^{*}\right)$. By continuity of $c(t(\mathbf{x}))$ there exist $\left(\tilde{x}_{1}, \tilde{x}_{2}\right) \in \mathcal{N}$ such that $\mathbf{z}\left(\tilde{x}_{1}, \tilde{x}_{2}, \psi\left(\tilde{x}_{1}, \tilde{x}_{2}\right)\right)=\mathbf{c}\left(t^{*}+s\right)$ for some $s$ with $0<|s|<h$. Thus, at the same time we should have $c_{1}\left(t^{*}+s\right)=c_{1}\left(t^{*}\right)$ because we are on a level set for $z_{1}$, and $c_{1}\left(t^{*}+s\right) \neq c_{1}\left(t^{*}\right)$, which leads to a contradiction. We hence showed that $c_{1}$ is constant implies also that $c_{2}, c_{3}$ are constants, that is $\mathbf{z}\left(x_{1}, x_{2}, \psi\left(x_{1}, x_{2}\right)\right)=\mathbf{z}(\hat{\mathbf{x}})=\mathbf{c}\left(t^{*}\right)$ for every $\left(x_{1}, x_{2}\right) \in \mathcal{N}$. This concludes the proof of the claim.

It remains to prove that for all $\mathbf{x} \in \mathcal{D}$, it holds $\Gamma\left(t^{*}\right) \cap \mathcal{U}_{\mathbf{x}} \subset \bar{\Omega}_{L}\left(t^{*}\right) \cap \bar{\Omega}_{R}\left(t^{*}\right) \cap \mathcal{U}_{\mathbf{x}}$, where again $t^{*} \in I_{T}$ is such that $\mathbf{x} \in \Gamma\left(t^{*}\right)$. Suppose first that there exists a neighbourhood $\mathcal{U}$ of $\mathbf{x}_{0} \in \Gamma\left(t^{*}\right)$ for some $t^{*} \in I_{T}$ such that

$$
\Omega_{A}\left(t^{*}\right) \cap \mathcal{U}=\varnothing \quad \text { or } \quad \Omega_{M}\left(t^{*}\right) \cap \mathcal{U}=\varnothing .
$$

This is $\mathbf{z}\left(\mathbf{x}_{0}\right)=\mathbf{c}\left(t^{*}\right)$, and $\mathbf{z}(\mathbf{x})=\mathbf{c}(s(\mathbf{x}))$ with $s(\mathbf{x})>t^{*}$ or $s(\mathbf{x})<t^{*}$ for every $\mathbf{x} \in \mathcal{U}$. We want to prove that either $\mathbf{z}$ is not differentiable in $\mathbf{x}_{0}$, or $\nabla \mathbf{z}\left(\mathbf{x}_{0}\right)=0$. Suppose not, then there exists $\beta_{j} \in \mathbb{R} \backslash\{0\}$ and a unit vector $\mathbf{v}_{j}$ such that $\nabla z_{j}\left(\mathbf{x}_{0}\right) \cdot \mathbf{v}_{j}=\beta_{j}$ for some $j=1,2,3$. Observe also that the differentiability of $\mathbf{z}$ implies the existence of $\delta_{j} \in(0,1)$ such that

$$
z_{j}\left(\mathbf{x}_{0}+\alpha \mathbf{v}_{j}\right)-z_{j}\left(\mathbf{x}_{0}\right)-\alpha \beta_{j}=\alpha r_{j}\left(\alpha \mathbf{v}_{j}\right), \quad \forall \alpha:|\alpha|<\delta_{j}
$$

for some continuous functions $r_{j}$ bounded in modulus by $\frac{\beta_{j}}{2}$. This implies that $z_{j}\left(\mathbf{x}_{0}+\alpha \mathbf{v}_{j}\right)-z_{j}\left(\mathbf{x}_{0}\right)$ has the same sign as $\alpha \beta_{j}$. Therefore, as $\mathbf{c}$ is simple, there exists an interval $\left(t_{\delta_{j}}, t^{*}\right)$ (or $\left.\left(t^{*}, t_{\delta_{j}}\right)\right)$ where $c_{j}(t)-c_{j}\left(t^{*}\right)$ is both strictly positive and strictly negative for every $t \in\left(t_{\delta_{j}}, t^{*}\right)$ (or in $\left(t^{*}, t_{\delta_{j}}\right)$ ), thus leading to a contradiction. Therefore, if $\mathbf{z}$ is differentiable at $\mathbf{x}_{0} \in \Gamma\left(t^{*}\right)$ and $\nabla \mathbf{z}\left(\mathbf{x}_{0}\right) \neq 0$, then $\mathbf{x}_{0} \in \bar{\Omega}_{A}\left(t^{*}\right) \cap \bar{\Omega}_{M}\left(t^{*}\right)$.

Now, by the coarea formula (2.15) with $g$ chosen to be the characteristic function of $\mathcal{D}$, we notice that

$$
0=\int_{\mathcal{Z}} \int_{f^{-1}(\boldsymbol{\xi}) \cap \Omega} g(\mathbf{s}) \mathrm{d} \mathscr{H}^{2}(\mathbf{s}) \mathrm{d} \mathscr{H}^{1}(\boldsymbol{\xi})
$$

and we deduce that $\mathbf{z}$ is differentiable with $\nabla \mathbf{z} \neq 0$ for $\mathscr{H}^{2}$-almost every $\mathbf{x} \in \Gamma(t)$ for almost every $t \in I_{T}$. Let us call $J_{T}$ the subset of $I_{T}$ such that $\mathbf{x} \in \mathcal{D} \mathscr{H}^{2}$-almost everywhere in $\Gamma(t)$ for every $t \in J_{T}$. By arguing as above for the set $\mathcal{C}$, the coarea formula implies that the set of $\mathrm{x} \in \Omega$ such that $\mathbf{z}(\mathbf{x}) \in \mathbf{c}\left(I_{T} \backslash J_{T}\right)$ has measure zero. We can hence focus without loss of generality on $\Gamma\left(t^{*}\right)$ for some $t^{*} \in J_{T}$. Suppose now that there does not exist a neighbourhood of $\mathbf{x}_{s} \in \Gamma\left(t^{*}\right)$ such that $\Gamma\left(t^{*}\right) \cap \mathcal{U} \subset \bar{\Omega}_{A}\left(t^{*}\right) \cap \bar{\Omega}_{M}\left(t^{*}\right)$. In this case, as $\Gamma\left(t^{*}\right)$ is closed in $\Omega$, there exists a neighbourhood $\mathcal{U}_{s}$ of $\mathbf{x}_{s}$ satisfying (4.27). However, as $\nabla \mathbf{z}$ exists and is non null $\mathscr{H}^{2}$ almost everywhere on $\Gamma\left(t^{*}\right)$, there exists $\mathbf{x}_{a} \in \Gamma\left(t^{*}\right) \cap \mathcal{U}_{s}, \mathbf{x}_{a} \in \mathcal{D}$, and which must hence be in $\bar{\Omega}_{A}\left(t^{*}\right) \cap \bar{\Omega}_{M}\left(t^{*}\right)$, thus leading to a contradiction. We have therefore proved that the constructed family of moving interfaces $\Gamma(t)$ satisfies the condition in Definition 4.1 (iii), which concludes the proof.

The following corollaries are straightforward consequences of the above theorem: 
Corollary 4.1. Let $\mathbf{y} \in W^{1, \infty}\left(\Omega ; \mathbb{R}^{3}\right)$ satisfy a regular moving mask approximation. Then, there exist $\mathbf{a} \in L^{\infty}\left(\Omega ; \mathbb{R}^{3}\right), \mathbf{n} \in L^{\infty}\left(\Omega ; \mathbb{S}^{2}\right)$ such that

$$
\nabla \mathbf{y}=\mathrm{Q}+\mathbf{a}(\mathbf{x}) \otimes \mathbf{n}(\mathbf{x}), \quad \text { a.e. } \mathbf{x} \in \Omega .
$$

Conversely, if $\mathbf{y} \in W^{1, \infty}\left(\Omega ; \mathbb{R}^{3}\right)$ satisfies (4.29) for some $\mathbf{Q} \in S O(3), \mathbf{a} \in L^{\infty}\left(\Omega ; \mathbb{R}^{3}\right), \mathbf{n} \in L^{\infty}\left(\Omega ; \mathbb{S}^{2}\right)$ and $\mathbf{z}(\Omega)$, with $\mathbf{z}(\mathbf{x})=\mathbf{y}(\mathbf{x})-\mathbf{Q} \mathbf{x}$, is contained in an absolutely continuous simple curve of finite length, then $\mathbf{y}$ satisfies a regular moving mask approximation.

Corollary 4.2. Let $T>0, \Gamma(t)$ be a family of moving interfaces and $\bar{\Omega}_{A}(t), \bar{\Omega}_{M}(t)$ be connected for every $t \in(0, T)$. Then, $\mathbf{Z} \in W^{1, \infty}\left(\Omega, \mathbb{R}^{3}\right)$ for each $t \in(0, T)$ satisfying (4.21) is equal to

$$
\mathbf{Z}(x, t)= \begin{cases}\mathbf{z}(x)+\mathbf{c}_{1}(t), & \text { in } \Omega_{M}(t), \\ \mathbf{c}_{2}(t), & \text { in } \Omega_{A}(t),\end{cases}
$$

for some $\mathbf{z} \in W^{1, \infty}\left(\Omega, \mathbb{R}^{3}\right)$ such that $\nabla \mathbf{z}=\mathbf{a} \otimes \mathbf{n}$ almost everywhere.

Proof. The statement follows directly from Theorem 4.1, the fact that $\mathbf{Z}(\mathbf{x}, t)$ is continuous in $\mathbf{x}$ for each $t$ and the hypothesis that $\bar{\Omega}_{A}(t), \bar{\Omega}_{M}(t)$ are connected.

Corollary 4.2 hence implies that, under the above hypotheses, for each $t \in I_{T}$, the interface $\Gamma(t)$ is a subset of $\left\{\mathbf{x} \in \Omega: \mathbf{z}(\mathbf{x})=\mathbf{c}_{2}(t)-\mathbf{c}_{1}(t)\right\}$, that is of a level set of $\mathbf{z}$. This also means that the image of $\mathbf{z}$ is a one-dimensional curve. However $\Gamma(t)$ does not need to coincide with the family of moving interfaces constructed in the proof of Theorem 4.1, even if $\mathbf{c}_{2}-\mathbf{c}_{1}$ is absolutely continuous, simple and of finite length. Indeed, in the proof of Theorem 4.1 the phase interfaces must be constructed as subsets of level sets for $\mathbf{z}$, but the construction of $\Omega_{A}(t), \Omega_{M}(t)$ is arbitrary and could be done differently. For example one could swap the definition of $\Omega_{A}(t)$ and $\Omega_{M}(t)$ in (4.23), or replace $s \in[0, t)$ and $s \in[0, t]$ in the definition of $\Omega_{M}$ and $\Omega_{A}$ respectively with $\left|s-t_{0}\right|<t$ and $s \in I_{T} \backslash\left[t_{0}-t, t_{0}+t\right]$ for some $t_{0} \in I_{T}$, thus getting a different family of moving interfaces.

The next corollary gives some information about the interface velocity. We define the normal velocity of $\Gamma\left(t^{*}\right)$ at time $t^{*} \in I_{T}$ and at $\mathbf{x} \in \Gamma\left(t^{*}\right)$, namely $(\mathbf{v} \cdot \mathbf{n})\left(\mathbf{x}, t^{*}\right)$, as $\dot{\gamma}\left(t^{*}\right) \cdot \mathbf{n}\left(\mathbf{x}, t^{*}\right)$, where $\mathbf{n}\left(\mathbf{x}, t^{*}\right)$ is the unit normal to $\Gamma\left(t^{*}\right)$ at $\mathbf{x} \in \Gamma\left(t^{*}\right)$, and $\gamma(t)$ is a generic absolutely continuous path differentiable at $t^{*}$ such that $\gamma(t) \in \Gamma(t)$ for each $t \in I_{T}$ and $\gamma\left(t^{*}\right)=\mathbf{x}$. Clearly $(\mathbf{v} \cdot \mathbf{n})\left(\mathbf{x}, t^{*}\right)$ is well defined if its value is independent of the choice of $\gamma$ among the admissible paths, and if $\mathbf{n}\left(\mathbf{x}, t^{*}\right)$ is well defined.

Corollary 4.3. Let $\mathbf{z} \in W^{1, \infty}\left(\Omega, \mathbb{R}^{3}\right)$ satisfy (4.22), $|a|>0$ a.e. in $\Omega$, and $\mathbf{z}(\Omega)$ be contained in the image of an absolutely continuous simple curve $\mathbf{c}: I_{T} \rightarrow \mathbb{R}^{3}$ of finite length. Assume further that $\Gamma(t)$ is the family of moving interfaces constructed in the proof of Theorem 4.1. Then, the normal velocity of $\Gamma(t)$ at a point $\mathbf{x} \in \Gamma(t)$, denoted $(\mathbf{v} \cdot \mathbf{n})(\mathbf{x}, t)$, satisfies

$$
\mathbf{a}(\mathbf{x})(\mathbf{v} \cdot \mathbf{n})(\mathbf{x}, t)=\dot{\mathbf{c}}(t), \quad \text { a.e. } t \in(0, T), \mathscr{H}^{2} \text {-a.e. } \mathbf{x} \in \Gamma(t) .
$$

Proof. By the coarea formula (2.15) with $g$ chosen to be the characteristic function of the set where $z$ is not differentiable and $|a|>0$, we notice that

$$
0=\int_{0}^{T} \int_{\mathbf{z}^{-1}(\mathbf{c}(t)) \cap \Omega} g(\mathbf{s}) \mathrm{d} \mathscr{H}^{2}(\mathbf{s})|\dot{\mathbf{c}}(t)| \mathrm{d} t .
$$


As the argument in the integral is non negative, we deduce that $\mathbf{z}$ is differentiable and $|\mathbf{a}|>0$ for $\mathscr{H}^{2}$-almost every $\mathbf{x} \in \Gamma(t)$ for almost every $t \in I_{T}$. As showed in the proof of Theorem $4.1 \mathbf{n}$ is well defined for all these $\mathbf{x}$. Let us consider $\gamma(t)$, an absolutely continuous path in $\Omega$ such that $\gamma(t) \in \Gamma(t)$ for every $t \in I_{T}$. We have

$$
\mathbf{z}(\gamma(t))=\mathbf{c}(t), \quad \forall t \in\left(t_{0}, t_{1}\right)
$$

for some $0 \leq t_{0}<t_{1} \leq T$. Taking the time derivative of this identity we get

$$
\dot{\mathbf{c}}(t)=\nabla \mathbf{z}(\gamma(t)) \dot{\gamma}(t)=\mathbf{a}(\gamma(t))(\dot{\gamma}(t) \cdot \mathbf{n}(\gamma(t), t))=\mathbf{a}(\mathbf{x})(\dot{\gamma}(t) \cdot \mathbf{n}(\mathbf{x}, t))
$$

which is the claimed result, as $(\dot{\gamma}(t) \cdot \mathbf{n}(\mathbf{x}, t))$ is independent of $\gamma$ chosen for a.e. $t \in I_{T}$, a.e. $\mathbf{x} \in$ $\Gamma(t)$.

Remark 4.6. An important consequence of the above corollary is that $\frac{\mathbf{a}(\mathbf{x})}{|\mathbf{a}(\mathbf{x})|}$ is constant $\mathscr{H}^{2}$ almost everywhere on $\Gamma(t)$ for almost every $t$. At the same time, there might be jumps in $|\mathbf{a}(\mathbf{x})|$ along a single interface and jumps for $\frac{\mathbf{a}(\mathbf{x})}{|\mathbf{a}(\mathbf{x})|}$ across interfaces.

Remark 4.7. If we assume the determinant of $\nabla \mathbf{y}=1+\nabla \mathbf{z}=1+\mathbf{a} \otimes \mathbf{n}$ to be a positive constant $\mathfrak{D}$ almost everywhere in $\Omega$, than we can deduce that on almost all interfaces $|\mathbf{a}(\mathbf{x})|$ can jump if and only if there is a jump in $\mathbf{n}(\mathbf{x})$. Indeed, this is a direct consequence of the following two facts: the first is that the direction of $\mathbf{a}(\mathbf{x})$ is fixed on almost all interfaces, the second is that, $\operatorname{det}(\nabla \mathbf{y})=\mathfrak{D}$ a.e. in $\Omega$ implies $\mathbf{a} \cdot \mathbf{n}=\mathfrak{D}-1$ a.e. in $\Omega$, and hence, by the coarea formula (see the argument in the proof of Corollary 4.3$), \mathbf{a} \cdot \mathbf{n}=\mathfrak{D}-1, \mathscr{H}^{2}$-almost everywhere on $\Gamma(t)$ for almost all $t$.

A different perspective on the velocity of $\Gamma(t)$ is given by

Corollary 4.4. Let $\mathbf{z} \in W^{1, \infty}\left(\Omega, \mathbb{R}^{3}\right)$ satisfy (4.22), $|\mathbf{a}|>0$ a.e. in $\Omega$, and $\mathbf{z}(\Omega)$ be contained in the image of an absolutely continuous simple curve $\mathbf{c}: I_{T} \rightarrow \mathbb{R}^{3}$ of finite length. Assume further that $\Gamma(t)$ is the family of moving interfaces constructed in the proof of Theorem 4.1. Then,

$$
\left\langle\dot{\chi} \Omega_{M}, \xi\right\rangle=|\dot{\mathbf{c}}(t)| \int_{\Gamma(t)} \frac{\xi(\mathbf{s})}{|\mathbf{a}(\mathbf{s})|} \mathrm{d} \mathscr{H}^{2}(\mathbf{s}), \quad \forall \xi \in C^{0}(\Omega), \text { a.e. } t \in(0, T) .
$$

Proof. We first notice that, by the coarea formula,

$$
\begin{aligned}
\int_{\Omega} \chi_{\Omega_{M}}(\mathbf{x}, t) \xi(\mathbf{x}) \mathrm{d} \mathbf{x}= & \int_{0}^{T} \int_{\mathbf{z}^{-1}(\mathbf{c}(\tau)) \cap \Omega_{A}(t)} \frac{\xi(\mathbf{s})}{|\mathbf{a}(\mathbf{s})|} \mathrm{d} \mathscr{H}^{2}(\mathbf{s})|\dot{\mathbf{c}}(\tau)| \mathrm{d} \tau \\
& =\int_{0}^{t} \int_{\mathbf{z}^{-1}(\mathbf{c}(\tau)) \cap \Omega} \frac{\xi(\mathbf{s})}{|\mathbf{a}(\mathbf{s})|} \mathrm{d} \mathscr{H}^{2}(\mathbf{s})|\dot{\mathbf{c}}(\tau)| \mathrm{d} \tau .
\end{aligned}
$$

Therefore,

$$
\frac{d}{d t} \int_{\Omega} \chi_{\Omega_{M}}(\mathbf{x}, t) \xi(\mathbf{x}) \mathrm{d} \mathbf{x}=|\dot{\mathbf{c}}(t)| \int_{\mathbf{z}^{-1}(\mathbf{c}(t)) \cap \Omega} \frac{\xi(\mathbf{s})}{|\mathbf{a}(\mathbf{s})|} \mathrm{d} \mathscr{H}^{2}(\mathbf{s}) .
$$

which is the claimed result. 


\section{Basic properties of microstructures}

According to the results of the previous sections, we can restrict our attention to deformation gradients satisfying for every $t \in(0, T)$

$$
\begin{cases}\nabla \mathbf{y}(\mathbf{x}, t)=1+\mathbf{a}(\mathbf{x}) \otimes \mathbf{n}(\mathbf{x}), & \text { a.e. } \mathbf{x} \in \Omega_{M}(t), \\ \nabla \mathbf{y}(\mathbf{x}, t)=1, & \text { a.e. } \mathbf{x} \in \Omega_{A}(t)\end{cases}
$$

for some $\mathbf{a}(\mathbf{x}) \in L^{\infty}\left(\Omega, \mathbb{R}^{3}\right), \mathbf{n}(\mathbf{x}) \in L^{\infty}\left(\Omega, \mathbb{S}^{2}\right)$ such that $\mathbf{n}(\mathbf{x})$ is normal to the austenite-martensite interface in $\mathbf{x}$ at a certain time $t^{*} \in(0, t)$. Here we assumed without loss of generality to have $\mathbf{Q}=1$ in Definition 3.1. In this way the martensitic macroscopic deformation gradient is a function of the moving, possibly curved, austenite-martensite interface during phase transition. In light of the above considerations, we assume that martensitic microstructures arising from austenite to martensite phase transitions are described by deformation gradients $\nabla \mathbf{y}$ of the form

$$
\nabla \mathbf{y}(\mathbf{x})=1+\mathbf{a}(\mathbf{x}) \otimes \mathbf{n}(\mathbf{x}), \quad \nabla \mathbf{y} \in K^{q c}, \quad \text { a.e. } \mathbf{x} \in \Omega .
$$

As the determinant is constant in $K$, it follows that

$$
\operatorname{det} \nabla \mathbf{y}(\mathbf{x})=\mathfrak{D} \quad \text { a.e. } \mathbf{x} \in \Omega,
$$

for some constant $\mathfrak{D}>0$. ( $(\mathrm{H} 1)$ and $(\underline{\mathrm{H} 2})$ imply

$$
\mathbf{a}(\mathbf{x}) \cdot \mathbf{n}(\mathbf{x})=\mathfrak{D}-1
$$

for a.e. $\mathbf{x} \in \Omega$, and

$$
\nabla \times\left(a_{i}(\mathbf{x}) \mathbf{n}(\mathbf{x})\right)=\mathbf{0} \quad \text { for each } i=1,2,3,
$$

in a weak sense.

In conclusion, in what follows we look at martensite microstructures as a part of the domain where (H1) and (H2) hold. We first begin with an estimate for the norm of $\mathbf{a}(\mathbf{x})$ :

Proposition 5.1. Let $\lambda_{\max }$ and $\lambda_{\min }$ be respectively the biggest and the smallest eigenvalues of the

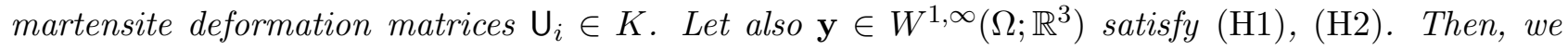
have

$$
|\mathfrak{D}-1| \leq|\mathbf{a}| \leq \lambda_{\max }-\lambda_{\min }, \quad \text { a.e. in } \Omega \text {. }
$$

Proof. The first inequality follows trivially from Cauchy-Schwarz and the fact that $\mathbf{a} \cdot \mathbf{n}=\mathfrak{D}-1$. In order to get the other one, we observe that by the polar decomposition theorem we have that

$$
\nabla \mathbf{y}(\mathbf{x})=\mathrm{R}(\mathbf{x}) \mathrm{F}(\mathbf{x})
$$

for almost every $\mathbf{x} \in \Omega$, where $\mathrm{R}(\mathbf{x}) \in S O(3)$ and $\mathrm{F}(\mathbf{x})$ is symmetric positive definite. The argument below holds for almost every $\mathbf{x} \in \Omega$. By arguing as in [5] one can deduce that, in order to have a rank-one connection with the identity matrix, the eigenvalues of $\mathrm{F}$, namely $\sigma_{\min } \leq \sigma_{\operatorname{mid}} \leq \sigma_{\max }$, must satisfy

$$
\sigma_{\text {mid }}=1, \quad \sigma_{\min } \sigma_{\max }=\mathfrak{D}
$$


Therefore, we have

$$
\operatorname{det} \mathrm{F}=\mathfrak{D}=\sigma_{\min } \sigma_{\max }, \quad \operatorname{tr}\left(\mathrm{F}^{T} \mathrm{~F}\right)=1+\sigma_{\min }^{2}+\sigma_{\text {max }}^{2}
$$

On the other hand,

$$
\nabla \mathbf{y}^{T} \nabla \mathbf{y}=\mathrm{F}^{T} \mathrm{~F}=1+\mathbf{a} \otimes \mathbf{n}+\mathbf{n} \otimes \mathbf{a}+|\mathbf{a}|^{2} \mathbf{n} \otimes \mathbf{n}
$$

which yields

$$
\operatorname{tr}\left(\mathrm{F}^{T} \mathrm{~F}\right)=3+2 \mathbf{a} \cdot \mathbf{n}+|\mathbf{a}|^{2}=1+2 \mathfrak{D}+|\mathbf{a}|^{2} .
$$

Therefore, by putting together (5.34) and (5.33) we deduce

$$
0 \leq|\mathbf{a}|^{2}=\left(\sigma_{\max }-\sigma_{\min }\right)^{2} \leq\left(\lambda_{\max }-\lambda_{\min }\right)^{2}
$$

Here we also made use of the following relation between eigenvalues proved in [5]:

$$
\lambda_{\min } \leq \sigma_{\min } \leq 1 \leq \sigma_{\max } \leq \lambda_{\max } .
$$

Another interesting property regards the divergence of $\mathbf{n} \otimes \mathbf{a}$ :

Proposition 5.2. Let $\mathbf{z} \in W^{1, \infty}\left(\Omega ; \mathbb{R}^{3}\right)$ be such that

$$
\nabla \mathbf{z}(\mathbf{x})=\mathbf{a}(\mathbf{x}) \otimes \mathbf{n}(\mathbf{x}), \quad \mathbf{a} \cdot \mathbf{n}=\mathfrak{D}-1 \in \mathbb{R}, \quad \text { a.e. in } \Omega .
$$

Then, $\nabla \cdot(\mathbf{n} \otimes \mathbf{a})=\mathbf{0}$ in the sense of distributions. Furthermore, if $\mathbf{n} \in W^{1,1}\left(\Omega ; \mathbb{R}^{3}\right)$ with $|\mathbf{n}|=1$ a.e. in $\Omega$, then $\nabla \cdot \mathbf{a}=0$ in the sense of distributions.

Proof. On the one hand, we have

$$
\nabla(\nabla \cdot \mathbf{z})=\nabla(\mathfrak{D}-1)=\mathbf{0}
$$

On the other hand

$$
\nabla(\nabla \cdot \mathbf{z})=\nabla \cdot(\nabla \mathbf{z})^{T}=\nabla \cdot(\mathbf{n} \otimes \mathbf{a})
$$

Here, both identities should be understood in the sense of distributions. By putting them together we hence get the first statement. As a consequence, if $\mathbf{n} \in W^{1,1}\left(\Omega ; \mathbb{R}^{3}\right)$ such that $|\mathbf{n}|=1$ a.e. in $\Omega$, we can write,

$$
\begin{aligned}
\int_{\Omega} \mathbf{a} \cdot \nabla \varphi \mathrm{d} \mathbf{x} & =\int_{\Omega} \mathbf{n}^{T} \mathbf{n} \otimes \mathbf{a} \cdot \nabla \varphi \mathrm{d} \mathbf{x} \\
& =\int_{\Omega} \mathbf{n} \otimes \mathbf{a}: \nabla(\mathbf{n} \varphi) \mathrm{d} \mathbf{x}-\int_{\Omega} \varphi \mathbf{n} \otimes \mathbf{a}: \nabla \mathbf{n} \mathrm{d} \mathbf{x} \\
& =-\frac{1}{2} \int_{\Omega} \varphi \mathbf{a} \cdot \nabla|\mathbf{n}|^{2} \mathrm{~d} \mathbf{x}=0
\end{aligned}
$$

for every $\varphi \in C_{c}^{\infty}(\Omega)$. 


\begin{tabular}{|c|c|c|c|c|c|c|}
\hline & & 1 & 1 & 1 & 1 & 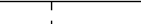 \\
\hline & & 1 & 1 & 1 & 1 & $-\frac{1}{2}$ \\
\hline$a=$ & $\mathbf{e}_{1}$ & 1 & 1 & I & 1 & $\mathbf{a}=-\mathbf{e}_{1}$ \\
\hline $\mathbf{n}=\mathbf{t}$ & $\mathbf{e}_{1}$ & i & i & i & ! & $\mathbf{n}=-\mathbf{e}_{1}$ \\
\hline 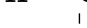 & & 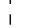 & 1 & 1 & 1 & $10 \frac{1}{1}$ \\
\hline I & & 1 & 1 & 1 & 1 & 1 \\
\hline I & & 1 & 1 & 1 & I & 1 \\
\hline & $\longmapsto$ & $\longmapsto$ & $\longmapsto$ & $\longleftarrow$ & $\longleftarrow$ & $\longleftarrow$ \\
\hline 1 & & 1 & 1 & I & I & I \\
\hline I & & 1 & 1 & I & I & 1 \\
\hline 1 & & 1 & 1 & 1 & 1 & 1 \\
\hline 1 & & 1 & 1 & 1 & 1 & 1 \\
\hline I & & 1 & 1 & I & I & 1 \\
\hline 1 & & 1 & I & 1 & 1 & 1 \\
\hline 1 & & 1 & 1 & I & 1 & $\Omega$ \\
\hline
\end{tabular}

Figure 2: Picture of two parallel interfaces moving in opposite directions and meeting at a certain interface where $\mathbf{a}, \mathbf{n}$ are discontinuous. In this case, $\nabla \cdot(\mathbf{n} \otimes \mathbf{a})=\mathbf{0}$, but $\nabla \cdot \mathbf{a} \neq 0$.

In general, it is not true that $\nabla \cdot \mathbf{a}=0$. Indeed, let us fix $\mathbf{e} \in \mathbb{R}^{3}$ with $|\mathbf{e}|=1$, and consider $\mathbf{z}$ to be of the form $\mathbf{z}(\mathbf{x})=\mathbf{e}(\mathbf{x} \cdot \mathbf{e})$. Let us also fix $c \in \mathbb{R}$ such that $\mathbf{x} \cdot \mathbf{e}=c$ for some $\mathbf{x} \in \Omega$ and define

$$
\mathbf{a}=\mathbf{n}= \begin{cases}\mathbf{e}, & \mathbf{x} \cdot \mathbf{e}<c, \\ -\mathbf{e}, & \mathbf{x} \cdot \mathbf{e}>c\end{cases}
$$

In this case, clearly $\mathbf{a} \cdot \mathbf{n}=1$ a.e. in $\Omega$, and $\nabla \cdot(\mathbf{n} \otimes \mathbf{a})=0$ in the distributional sense. However, $\mathbf{a} \in B V\left(\Omega ; \mathbb{R}^{3}\right)$ satisfies

$$
\nabla \mathbf{a}=-2 \mathbf{e} \otimes \mathbf{e} \mathscr{H}^{2} \mathrm{~L}\{\mathbf{x}: \mathbf{x} \cdot \mathbf{e}=c\}
$$

and, as $|\mathbf{e}|=1$ by hypothesis, $\nabla \cdot \mathbf{a} \neq 0$ in the distributional sense.

Keeping in mind the counterexample above, we extend the validity of the identity $\nabla \cdot \mathbf{a}=0$ in the following Corollary 5.1. In this result we use the space of special functions with bounded variation on $\Omega$, namely $S B V(\Omega)$. If $\varphi \in S B V(\Omega)$, its gradient is the sum of two Radon measures, one absolutely continuous with respect to the Lebesgue measure $\mathscr{L}^{3}$, and the other concentrated on a 2-rectifiable set $S_{\varphi}$, usually called the jump set. We denote by $\varphi^{-}, \varphi^{+}$the trace of $\varphi$ on the two sides of the jump set $S_{\varphi}$ respectively. We refer the interested reader to [2] and [20] for more details on this space.

Corollary 5.1. Let $\mathbf{z} \in W^{1, \infty}\left(\Omega ; \mathbb{R}^{3}\right)$ be such that

$$
\nabla \mathbf{z}(\mathbf{x})=\mathbf{a}(\mathbf{x}) \otimes \mathbf{n}(\mathbf{x}), \quad \mathbf{a} \cdot \mathbf{n}=\mathfrak{D}-1 \in \mathbb{R}, \quad \text { a.e. in } \Omega .
$$

Let $\mathbf{a}, \mathbf{n} \in S B V\left(\Omega ; \mathbb{R}^{3}\right) \cap L^{\infty}\left(\Omega ; \mathbb{R}^{3}\right),|\mathbf{n}|=1$ a.e. in $\Omega$ and let

$$
\begin{gathered}
\mathbf{n}^{-}(\mathbf{x}) \neq-\mathbf{n}^{+}(\mathbf{x}), \quad \text { if } \mathfrak{D} \neq 1, \\
\mathbf{a}^{-}(\mathbf{x})=-\mathbf{a}^{+}(\mathbf{x}) \Rightarrow \mathbf{n}^{-}(\mathbf{x}) \neq-\mathbf{n}^{+}(\mathbf{x}), \quad \text { if } \mathfrak{D}=1,
\end{gathered}
$$

for $\mathscr{H}^{2}$-a.e. $\mathbf{x} \in S_{\mathbf{n}} \cap S_{\mathbf{a}}$. Then, $\nabla \cdot \mathbf{a}=0$ in the sense of distributions.

The following lemma is needed for the proof of Corollary 5.1;

Lemma 5.1. Let $\varphi \in H^{1}\left(\Omega, \mathbb{R}^{3}\right)$, with $\nabla \varphi \in B V\left(\Omega ; \mathbb{R}^{3 \times 3}\right)$. Then, there exists $\mathbf{b}(\mathbf{x}) \in L^{1}\left(S_{\nabla \varphi} ; \mathbb{R}^{3}\right)$ such that

$$
(\nabla \boldsymbol{\varphi})^{+}(\mathbf{x})-(\nabla \boldsymbol{\varphi})^{-}(\mathbf{x})=\mathbf{b}(\mathbf{x}) \otimes \mathbf{m}(\mathbf{x}), \quad \mathscr{H}^{2} \text {-a.e. } \mathbf{x} \in S_{\nabla \varphi},
$$

with $\mathbf{m}(\mathbf{x})$ being the normal to $S_{\nabla \varphi}$ in $\mathbf{x}$. 
Proof. The proof of this type of result is usually done via a blow up argument and exploits continuity. This may be possible here, but we use a slightly different proof. Let $\varphi \in H^{1}\left(\Omega ; \mathbb{R}^{3}\right)$ and let $A$ be a Lipschitz open subset of $\Omega$. Since $\nabla \times \nabla \varphi=0$ in the sense of distribution, we have that the following integration by parts formula holds (see [23, Ch. 2, (2.18)])

$$
\int_{A} \nabla \varphi_{i} \cdot \nabla \times \boldsymbol{\psi} \mathrm{d} \mathbf{x}=\int_{\partial A}\left(\nabla \varphi_{i} \times \mathbf{m}\right) \cdot \boldsymbol{\psi} \mathrm{d} \mathscr{H}^{2}, \quad \forall \boldsymbol{\psi} \in H^{1}\left(\Omega ; \mathbb{R}^{3}\right),
$$

with $\mathbf{m}$ being the outpointing normal to $A$. We remark that, as stated in [23, Ch. 2, Thm 2.5], $\left(\nabla \varphi_{i} \times \mathbf{m}\right)$ is a well defined object in $H^{-\frac{1}{2}}(\partial A)$, and the integral on the right hand side should be interpreted as $\left\langle\nabla \varphi_{i} \times \mathbf{m}, \boldsymbol{\psi}\right\rangle_{H^{-\frac{1}{2}}, H^{\frac{1}{2}}}$. Let now $S$ be a Lipschitz 2-graph contained in $\Omega$ with normal $\mathbf{m}$ and let $U_{i}$ be a countable set of open neighbourhoods such that $U_{i} \subset \Omega, U_{i} \backslash S$ has exactly two connected components, namely $U_{i}^{+}$and $U_{i}^{-}$, and

$$
\mathscr{H}^{2}\left(S \backslash \bigcup U_{i}\right)=0
$$

We now define $\left(\nabla \varphi_{i} \times \mathbf{m}\right)^{ \pm}$to be the objects of $H^{-\frac{1}{2}}(S)$ satisfying (5.38) respectively for $A=U_{i}^{ \pm}$. From (5.38) we deduce

$$
\begin{aligned}
& 0=\int_{U_{i}} \nabla \varphi_{i} \cdot \nabla \times \boldsymbol{\psi} \mathrm{d} \mathbf{x}=\int_{U_{i}^{+}} \nabla \varphi_{i} \cdot \nabla \times \boldsymbol{\psi} \mathrm{d} \mathbf{x}+\int_{U_{i}^{-}} \nabla \varphi_{i} \cdot \nabla \times \boldsymbol{\psi} \mathrm{d} \mathbf{x} \\
&=\int_{S \cap U_{i}}\left(\left(\nabla \varphi_{i} \times \mathbf{m}\right)^{+}-\left(\nabla \varphi_{i} \times \mathbf{m}\right)^{-}\right) \cdot \boldsymbol{\psi} \mathrm{d} \mathscr{H}^{2},
\end{aligned}
$$

for every $\psi \in C_{c}^{\infty}\left(U_{i} ; \mathbb{R}^{3}\right)$. By repeating this argument on all $U_{i}$, this implies

$$
\left\|\left(\nabla \varphi_{i} \times \mathbf{m}\right)^{+}-\left(\nabla \varphi_{i} \times \mathbf{m}\right)^{-}\right\|_{H^{-\frac{1}{2}(S)}}=0, \quad i=1,2,3
$$

which is a weak Hadamard jump condition for $H^{1}\left(\Omega ; \mathbb{R}^{3}\right)$ on Lipschitz 2-graphs. Furthermore, if $\nabla \varphi \in S B V(\Omega)$, we know that the set where it is discontinuous, namely $S_{\nabla \varphi}$ is 2-rectifiable (see e.g., [2]). Therefore, by Proposition (2.4) we can cover $S_{\nabla \varphi}$ with countably many Lipschitz graphs where (5.39) holds. On the other hand, from [2, Thm 3.77] we know that the trace of $\nabla \varphi_{i}$ is well defined for almost every point of $S_{\nabla \varphi}$. Collecting these two facts we thus deduce the desired result.

Remark 5.1. Equation (5.39) is a very weak version of the Hadamard jump condition on Lipschitz surfaces $\Gamma$ with normal $\mathbf{m}$ for functions $\mathbf{y} \in H^{1}\left(\Omega ; \mathbb{R}^{3}\right)$. Indeed, we can only make sense to the tangential trace $\nabla \mathbf{y} \times \mathbf{m}$ of $\mathbf{y}$ on $\Gamma$ as an object of $H^{-\frac{1}{2}}(\Gamma)$, and (5.39) states that, across $\Gamma, \nabla \mathbf{y} \times \mathbf{m}$ must not jump as an object of $H^{-\frac{1}{2}}(\Gamma)$, which is kind of an average sense.

Proof. It follows from the definition of jump points of a $B V$ function (see e.g., [2, 20]) that (5.35) and $|\mathbf{n}|=1$ hold $\mathscr{H}^{2}$-almost everywhere on $S_{\mathbf{a}} \cup S_{\mathbf{n}}$. Therefore, under our hypotheses (5.36)-(5.37) $\mathbf{a} \otimes \mathbf{n} \in S B V(\Omega) \cap L^{\infty}(\Omega)$ and $S_{\mathbf{a} \otimes \mathbf{n}}=S_{\mathbf{a}} \cup S_{\mathbf{n}}$ up to an $\mathscr{H}^{2}$-negligible set. Here, a, $\mathbf{n}$ are chosen to be the precise representatives for a,n. Furthermore, Lemma 5.1 implies that a Hadamard jump condition must hold across $S_{\mathbf{a} \otimes \mathbf{n}}$, so that

$$
\mathbf{a}^{+} \otimes \mathbf{n}^{+}-\mathbf{a}^{-} \otimes \mathbf{n}^{-}=\mathbf{b} \otimes \mathbf{m}, \quad \mathscr{H}^{2} \text {-a.e. on } S_{\mathbf{a} \otimes \mathbf{n}},
$$

for some $\mathbf{b} \in L^{\infty}\left(S_{\mathbf{a} \otimes \mathbf{n}} ; \mathbb{R}^{3}\right)$ and with $\mathbf{m}$ being the normal to $S_{\mathbf{a} \otimes \mathbf{n}}$. In case $\mathfrak{D} \neq 1$, this, together with (5.36) and $|\mathbf{n}|=1$, imply that the only possible scenarios are the following on $S_{\mathbf{a}} \cup S_{\mathbf{n}}$ : 
(a) $\mathbf{n}^{+}=\mathbf{n}^{-}=\mathbf{m}$, in which case $\mathbf{b}=\mathbf{a}^{+}-\mathbf{a}^{-}$;

(b) $\mathbf{a}^{+}=\xi \mathbf{a}^{-}$, in which case $\mathbf{m} \| \xi \mathbf{n}^{+}-\mathbf{n}^{-}$and $\mathbf{b}\left\|\mathbf{a}^{+}\right\| \mathbf{a}^{-}$

for some $\xi \in L^{\infty}\left(S_{\mathbf{n}}\right)$. Taking the trace of (5.40) implies also $\mathbf{b} \cdot \mathbf{m}=0$. As a consequence, by (国) -(逐) $\left(\mathbf{a}^{+}-\mathbf{a}^{-}\right) \cdot \mathbf{m}=0 \mathscr{H}^{2}$-a.e. on $S_{\mathbf{a}} \cup S_{\mathbf{n}}$, that is, the divergence of $\mathbf{a}$ has no singular part. In case $\mathfrak{D}=1$, (5.37) allows also to have $\mathbf{n}^{+}=-\mathbf{n}^{-}=\mathbf{m}$ in $S_{\mathbf{a}}$, when $\mathbf{a}^{+} \neq-\mathbf{a}^{-}$. In which case $\left(\mathbf{a}^{+}-\mathbf{a}^{-}\right) \cdot \mathbf{m}=0$ follows just by the fact that $\mathbf{a} \cdot \mathbf{n}=0$.

It just remains to check that the part of $\nabla \cdot \mathbf{a}$ which is absolutely continuous with respect to $\mathscr{L}^{3}$ is null as well. To this aim, we first observe that, by the chain rule for $B V$ functions (see e.g., [2, Example 3.97])

$$
\mathbf{0}=\nabla \cdot(\mathbf{n} \otimes \mathbf{a})=\left(\mathbf{n}\left(\nabla^{a} \cdot \mathbf{a}\right)+\nabla^{a} \mathbf{n a}\right) \mathscr{L}^{3}+\left(\mathbf{n}^{+} \otimes \mathbf{a}^{+}-\mathbf{n}^{-} \otimes \mathbf{a}^{-}\right) \mathbf{m} \mathscr{H}^{2} \mathbf{L} S_{\mathbf{a} \otimes \mathbf{n}},
$$

where we denoted by $\nabla^{a}$ the absolutely continous part of the gradient. Given (a) $-(\mathrm{b}$ ) above, under our hypotheses we have $\left(\mathbf{n}^{+} \otimes \mathbf{a}^{+}-\mathbf{n}^{-} \otimes \mathbf{a}^{-}\right) \mathbf{m}=\mathbf{0} \mathscr{H}^{2}$-a.e. on $S_{\mathbf{a} \otimes \mathbf{n}}$. Furthermore, as $|\mathbf{n}|=1$ a.e., we have

$$
\mathbf{0}=\nabla|\mathbf{n}|^{2}=\mathbf{n}^{T} \nabla^{a} \mathbf{n} \mathscr{L}^{3} .
$$

Therefore, multiplying (5.41) by $\mathbf{n}$ and exploiting the fact that $|\mathbf{n}|=1$ a.e., we thus get

$$
\nabla^{a} \cdot \mathbf{a}=0, \quad \text { a.e. in } \Omega .
$$

Therefore, as $\mathbf{a} \in S B V(\Omega)$, for every $\phi \in C_{c}^{1}(\Omega)$ we have

$$
-\int_{\Omega} \nabla \varphi \cdot \mathbf{a} \mathrm{d} \mathbf{x}=\int_{S_{\mathbf{a}}} \varphi\left(\mathbf{a}^{+}-\mathbf{a}^{-}\right) \cdot \mathbf{m} \mathrm{d} \mathscr{H}^{2}+\int_{\Omega} \varphi \nabla^{a} \cdot \mathbf{a} \mathrm{d} \mathbf{x}=0
$$

which concludes the proof.

We now focus on compound twins. Thanks to Proposition 2.3 we know that if two martensite variants $\mathrm{U}_{1}, \mathrm{U}_{2} \in \mathbb{R}_{S y \mathrm{~m}^{+}}^{3 \times 3}$ form a compound twin, then there exists $\mu>0, \mathbf{v} \in \mathbb{S}^{2}$ such that $\mathrm{U}_{1} \mathbf{v}=\mathrm{U}_{2} \mathbf{v}=\mu \mathbf{v}$. In this case, [17, Theorem 2.5.1] states:

Theorem 5.1. Let $\mathrm{U}_{1}, \ldots, \mathrm{U}_{n} \in \mathbb{R}_{\text {Sym }}^{3 \times 3}$, such that $\operatorname{det} \mathrm{U}_{i}=\mathfrak{D}>0$, and such that $\mathrm{U}_{i} \mathbf{v}=\mu \mathbf{v}$ for some $\mu>0, \mathbf{v} \in \mathbb{S}^{2}$, for each $i=1, \ldots, n$. Let also

$$
H=\bigcup_{i=1}^{n} S O(3) \mathrm{U}_{i}
$$

Then, there exists $l \in \mathbb{N}, \mathbf{w}_{1}, \ldots, \mathbf{w}_{l}$ such that

$$
H^{q c}=\left\{\mathrm{F} \in \mathbb{R}^{3 \times 3} \mid \begin{array}{c}
\operatorname{det} \mathrm{F}=\mathfrak{D}, \mathrm{F}^{T} \mathrm{~F} \mathbf{v}=\mu^{2} \mathbf{v} \text { and } \\
\left|\mathrm{F} \mathbf{w}_{i}\right|^{2} \leq \max _{j=1, \ldots, n}\left|\mathrm{U}_{j} \mathbf{w}_{i}\right|^{2} \text { for each } i=1, \ldots, l .
\end{array}\right\}
$$

Therefore, in this simple case which includes compound twins, we can actually construct the quasiconvex hull of the set. An interesting result is given by the following lemma: 
Lemma 5.2. Let $\mathrm{U}_{1}, \ldots, \mathrm{U}_{n} \in \mathbb{R}_{\text {Sym }}^{3 \times 3}$, such that $\operatorname{det} \mathrm{U}_{i}=\mathfrak{D}>0$, and such that $\mathrm{U}_{i} \mathbf{v}=\mu \mathbf{v}$ for some $\mu>0, \mathbf{v} \in \mathbb{S}^{2}$, for each $i=1, \ldots, n$. Let also

$$
H=\bigcup_{i=1}^{n} S O(3) \mathrm{U}_{i},
$$

and $\mu \neq 1$. Then every map $\mathbf{y} \in W^{1, \infty}\left(\Omega ; \mathbb{R}^{3}\right)$ satisfying $\nabla \mathbf{y}(\mathbf{x}) \in H^{q c}$ and $\nabla \mathbf{y}(\mathbf{x})=1+\mathbf{a}(\mathbf{x}) \otimes \mathbf{n}(\mathbf{x})$ for a.e. $\mathbf{x}$ in $\Omega$ is such that $\mathbf{a} \otimes \mathbf{n}$ is constant, and $|\mathbf{a}|=|\mu|^{-1}\left|\mathfrak{D}-\mu^{2}\right|$.

Remark 5.2. It comes up in the proof of Lemma 5.2 that the following condition

$$
1 \geq \frac{\mu^{2}\left(1-\mu^{2}\right)}{\left(\mathfrak{D}^{2}-\mu^{4}\right)}>0
$$

is necessary in order to have the existence of $\mathbf{a} \in \mathbb{R}^{3}, \mathbf{n} \in \mathbb{S}^{2}$ such that $1+\mathbf{a} \otimes \mathbf{n} \in H^{q c}$. We refer the reader to [5] for some stricter necessary conditions for this to hold.

Proof. Thanks to a change of coordinates, we can suppose without loss of generality that $\mathbf{v}=\mathbf{e}_{3}$. In this case, every $\mathrm{F} \in H^{q c}$ satisfies

$$
\mathbf{F}^{T} \mathbf{F}=\left[\begin{array}{ccc}
\alpha & \gamma & 0 \\
\gamma & \beta & 0 \\
0 & 0 & \mu^{2}
\end{array}\right]
$$

and

$$
\alpha \beta-\gamma^{2}=\mathfrak{D}^{2} \mu^{-2} .
$$

We are first interested in solving $1+\mathbf{a} \otimes \mathbf{n} \in H^{q c}$ for $\mathbf{a} \in \mathbb{R}^{3}, \mathbf{n} \in \mathbb{S}^{2}$. By (15.43), the first step is to solve the following nonlinear system of equations for the components of $\mathbf{a}$ and $\mathbf{n}$ :

$$
\begin{cases}1+2 a_{1} n_{1}+|a|^{2} n_{1}^{2} & =\alpha \\ 1+2 a_{2} n_{2}+|a|^{2} n_{2}^{2} & =\beta \\ a_{1} n_{2}+a_{2} n_{1}+|a|^{2} n_{1} n_{2} & =\gamma \\ a_{3} n_{1}+a_{1} n_{3}+|a|^{2} n_{1} n_{3} & =0 \\ a_{3} n_{2}+a_{2} n_{3}+|a|^{2} n_{2} n_{3} & =0 \\ 1+2 a_{3} n_{3}+|a|^{2} n_{3}^{2} & =\mu^{2}\end{cases}
$$

subject to the constraint (5.44). As a first step we compute $\alpha \beta-\gamma^{2}$ using system (5.45). After rearranging terms

$$
\alpha \beta-\gamma^{2}=2\left(a_{1} n_{1}+a_{2} n_{2}\right)+1+|\mathbf{a}|^{2}\left(n_{1}^{2}+n_{2}^{2}\right)-\left(a_{1} n_{2}-a_{2} n_{1}\right)^{2} .
$$

Since $|\mathbf{n}|=1$, using last equation of (5.45) follows that

$$
|\mathbf{a}|^{2}\left(n_{1}^{2}+n_{2}^{2}\right)=|\mathbf{a}|^{2}\left(1-n_{3}^{2}\right)=|\mathbf{a}|^{2}+1+2 a_{3} n_{3}-\mu^{2},
$$

which leads to

$$
\alpha \beta-\gamma^{2}=2(a \cdot n)+2+|\mathbf{a}|^{2}-\mu^{2}-\left(a_{1} n_{2}-a_{2} n_{1}\right)^{2} .
$$


Finally, by recalling (5.31) we deduce

$$
\alpha \beta-\gamma^{2}=2 \mathfrak{D}+|\mathbf{a}|^{2}-\mu^{2}-\left(a_{1} n_{2}-a_{2} n_{1}\right)^{2} .
$$

Thus, it is immediately seen that (5.46) together with the first of (5.44) implies

$$
\left(a_{1} n_{2}-a_{2} n_{1}\right)^{2}=2 \mathfrak{D}+|\mathbf{a}|^{2}-\mu^{2}-\frac{\mathfrak{D}^{2}}{\mu^{2}}=|\mathbf{a}|^{2}-\frac{1}{\mu^{2}}\left(\mathfrak{D}-\mu^{2}\right)^{2} .
$$

On the other hand, exploiting the last equation of (5.45) in the fourth and fifth one we have

$$
\begin{aligned}
& a_{2} n_{3}-a_{3} n_{2}=-\frac{n_{2}}{n_{3}}\left(\mu^{2}-1\right) \\
& a_{3} n_{1}-a_{1} n_{3}=\frac{n_{1}}{n_{3}}\left(\mu^{2}-1\right) .
\end{aligned}
$$

We note that it is legitimate to divide by $n_{3}$ since the last identity in (5.45) together with $\mu \neq 1$ implies $n_{3} \neq 0$. By putting together (5.47)-(5.49) we thus get

$$
\mathbf{a} \times \mathbf{n}=\left[\begin{array}{c}
-\frac{n_{2}}{n_{3}}\left(\mu^{2}-1\right) \\
\frac{n_{1}}{n_{3}}\left(\mu^{2}-1\right) \\
\pm \sqrt{|\mathbf{a}|^{2}-\frac{1}{\mu^{2}}\left(\mathfrak{D}-\mu^{2}\right)^{2}}
\end{array}\right] .
$$

Since $(\mathbf{a} \times \mathbf{n}) \cdot \mathbf{n}=0$ we have

$$
n_{3} \sqrt{|\mathbf{a}|^{2}-\frac{1}{\mu^{2}}\left(\mathfrak{D}-\mu^{2}\right)^{2}}=0,
$$

which, as $n_{3} \neq 0$, leads to $|\mathbf{a}|^{2}=\frac{1}{\mu^{2}}\left(\mathfrak{D}-\mu^{2}\right)^{2}$. In this case the norm of $\mathbf{a}$ is hence forced to be constant. We restrict ourselves without loss of generality to the case $\mathfrak{D} \neq \mu^{2}$.

We now want to write a in terms of the orthogonal vectors $\left(\mathbf{n}, \mathbf{n}^{\perp}, \mathbf{n} \times \mathbf{n}^{\perp}\right)$, where

$$
\mathbf{n}^{\perp}:=\left[\begin{array}{c}
n_{2} \\
-n_{1} \\
0
\end{array}\right], \quad \mathbf{n} \times \mathbf{n}^{\perp}=\left[\begin{array}{c}
n_{1} n_{3} \\
n_{2} n_{3} \\
n_{3}^{2}-1
\end{array}\right] .
$$

It is important to remark that, if $n_{1}=n_{2}=0$ then it is easy to see from (5.45) that $a_{1}=a_{2}=0$, so we do not lose any generality with this representation. We have,

$$
\mathbf{a}=a^{(1)} \mathbf{n}+a^{(2)} \mathbf{n}^{\perp}+a^{(3)} \mathbf{n} \times \mathbf{n}^{\perp} .
$$

As a first thing, recalling that $\operatorname{det}(1+\mathbf{a} \otimes \mathbf{n})=\mathfrak{D}$, we deduce that $a^{(1)}=\mathbf{a} \cdot \mathbf{n}=\mathfrak{D}-1$. On the other hand, taking cross product of $\mathbf{a}$ with $\mathbf{n}$ follows that

$$
\mathbf{n} \times \mathbf{a}=a^{(2)} \mathbf{n} \times \mathbf{n}^{\perp}-a^{(3)} \mathbf{n}^{\perp}
$$

A comparison of (5.53) with (5.50) and (5.51) thus leads to $a^{(3)}=\frac{1}{n_{3}}\left(1-\mu^{2}\right)$, and $a^{(2)}=0$, which implies

$$
\mathbf{a}=(\mathfrak{D}-1) \mathbf{n}+\frac{1}{n_{3}}\left(1-\mu^{2}\right) \mathbf{n} \times \mathbf{n}^{\perp}=\left(\mathfrak{D}-\mu^{2}\right) \mathbf{n}-\frac{1}{n_{3}}\left(1-\mu^{2}\right) \mathbf{e}_{3},
$$


with $\mathbf{e}_{3}=(0,0,1)^{T}$. As a first thing now we want to check whether it is possible to have $|\mathbf{a}|^{2}=$ $\frac{1}{\mu^{2}}\left(\mathfrak{D}-\mu^{2}\right)^{2}$. Since the orthogonal vectors $\mathbf{n}$ and $\mathbf{n} \times \mathbf{n}^{\perp}$ satisfy $|\mathbf{n}|=1$ and $\left|\mathbf{n} \times \mathbf{n}^{\perp}\right|^{2}=1-n_{3}^{2}$, by rearranging the terms it is possible to obtain

$$
n_{3}^{2}=\frac{\left(1-\mu^{2}\right)^{2}}{\left(1-\mu^{2}\right)^{2}+\frac{1}{\mu^{2}}\left(\mu^{2}-\mathfrak{D}\right)^{2}-(\mathfrak{D}-1)^{2}}=\frac{\left(1-\mu^{2}\right)}{\frac{1}{\mu^{2}}\left(\mathfrak{D}^{2}-\mu^{4}\right)} .
$$

It is easy to check that a pair of vectors $(\mathbf{n}, \mathbf{a})$ with a defined in terms of $\mathbf{n}$ as in (5.54), and where $n_{3}$ is given by (5.55), satisfies the equations of (5.45). Thus, it turns out that (5.42) in necessary in order not to contradict $|\mathbf{n}|=1,1-n_{3}^{2}=n_{1}^{2}+n_{2}^{2} \geq 0$ and $n_{3}^{2}>0$ (see also Remark [5.2).

Let us now check when a map $\mathbf{y} \in W^{1, \infty}\left(\Omega ; \mathbb{R}^{3}\right)$ such that $\nabla \mathbf{y}(\mathbf{x})$ satisfies (5.43)-(5.44) and $\nabla \mathbf{y}(\mathbf{x})=$ $1+\mathbf{a}(\mathbf{x}) \otimes \mathbf{n}(\mathbf{x})$ for a.e. $\mathbf{x}$ in $\Omega$. We have to verify that conditions expressed in (5.32) hold, that is, we have to check when the constructed deformations are actually gradients, by verifying that $\nabla \times\left(a_{i} \mathbf{n}\right)=0$ for $i=1,2,3$, in the distributional sense. Since $\mathfrak{D}$ and $\mu$ are constants, so is $\left|n_{3}\right|=$ $\sqrt{\left(\mu^{2}-\mu^{4}\right)\left(\mathfrak{D}^{2}-\mu^{4}\right)^{-1}}$. Hence, by (5.54),$\left|a_{3}\right|$ is constant and non zero as long as $\mu^{2} \neq \mathfrak{D}$ and $\mu \neq 1$, which is our case. Furthermore, $n_{3}=\operatorname{sgn}\left(n_{3}\right)\left|n_{3}\right|$ and $a_{3}= \pm\left|a_{3}\right| \operatorname{sgn}\left(n_{3}\right)$, where the sign depends on $\mu, \mathfrak{D}$ only and is hence fixed. We can hence suppose without loss of generality $a_{3}=\left|a_{3}\right| \operatorname{sgn}\left(n_{3}\right)$. Therefore,

$$
\nabla \times\left(a_{3} \mathbf{n}\right)=\left|a_{3}\right| \nabla \times\left(\operatorname{sgn}\left(n_{3}\right) \mathbf{n}\right)=0
$$

implies

$$
\nabla \times\left(\operatorname{sgn}\left(n_{3}\right) \mathbf{n}\right)=0
$$

in the sense of distributions. This implies the existence of $\psi \in W^{1,2}(\Omega)$ such that $\operatorname{sgn}\left(n_{3}\right) \mathbf{n}=\nabla \psi($ see e.g., [23]). On the other hand, by Proposition 5.2

$$
\nabla \cdot\left(\mathbf{a} n_{3}\right)=\left|n_{3}\right| \nabla \cdot\left(\operatorname{sgn}\left(n_{3}\right) \mathbf{a}\right)=0,
$$

which implies

$$
\nabla \cdot\left(\operatorname{sgn}\left(n_{3}\right) \mathbf{a}\right)=0
$$

in the sense of distributions. Furthermore, we have from (5.54) that

$$
\nabla \cdot\left(\operatorname{sgn}\left(n_{3}\right) \mathbf{a}\right)=\left(\mathfrak{D}-\mu^{2}\right) \nabla \cdot\left(\operatorname{sgn}\left(n_{3}\right) \mathbf{n}\right)
$$

which thus implies that $\psi$ is harmonic in the sense of distributions. By using standard elliptic theory (see e.g., [19]) we can thus deduce that $\psi \in C^{\infty}(\Omega)$. On the other hand, as $|\nabla \psi|^{2}=1$, we have

$$
\nabla \psi^{T} \nabla^{2} \psi=0, \quad \text { for all } \mathbf{x} \in \Omega
$$

which implies that one eigenvalue of the Hessian matrix is null. Another eigenvalue is 0 as $\mathbf{e}_{3}$ is a left eigenvector related to 0 for $\nabla^{2} \psi$, and is not parallel to $\mathbf{n}$ unless $\mathbf{n}=\mathbf{e}_{3}$, in which case (5.55) forces $\operatorname{sgn}\left(n_{3}\right) \mathbf{n}=\mathbf{e}_{3}$ everywhere. The fact that $\psi$ is harmonic, i.e., $\operatorname{tr} \nabla^{2} \psi=0$, thus means that $\nabla \psi$ is constant. Therefore, $\operatorname{sgn}\left(n_{3}\right) \mathbf{n}$ is constant, and as a consequence of (15.54), so is $\operatorname{sgn}\left(n_{3}\right) \mathbf{a}$ and $\mathbf{a} \otimes \mathbf{n}$. 
Remark 5.3. In case $\mu=1$, it is not possible to deduce the same rigidity as in Lemma [5.2. Indeed, it can be deduced from equations (5.45) that $\mu=1$ implies either $n_{3}=a_{3}=0$, or $\mathbf{a}=(\mathfrak{D}-1) \mathbf{n}$, but in the latter case the last equation in (5.45) implies $\mathbf{a} \otimes \mathbf{n}=0$. The problem becomes thus two-dimensional, and we can rewrite

$$
\mathbf{n}=\left(n_{1}, n_{2}, 0\right)^{T}, \quad \mathbf{n}^{\perp}=\left(n_{2},-n_{1}, 0\right)^{T}, \quad \mathbf{a}(s)=(\mathfrak{D}-1) \mathbf{n}+s \mathbf{n}^{\perp},
$$

for some $s \in \mathbb{R}, n_{1}, n_{2} \in[-1,1]$ with $n_{1}^{2}+n_{2}^{2}=1$. Now, take two martensite variants, for example,

$$
\mathrm{U}_{1}=\frac{1}{2}\left[\begin{array}{ccc}
\lambda_{m}+\lambda_{M} & \lambda_{m}-\lambda_{M} & 0 \\
\lambda_{m}-\lambda_{M} & \lambda_{m}+\lambda_{M} & 0 \\
0 & 0 & 2
\end{array}\right], \quad \mathrm{U}_{2}=\frac{1}{2}\left[\begin{array}{ccc}
\lambda_{m}+\lambda_{M} & \lambda_{M}-\lambda_{m} & 0 \\
\lambda_{M}-\lambda_{m} & \lambda_{m}+\lambda_{M} & 0 \\
0 & 0 & 2
\end{array}\right]
$$

generating a compound twin, and such that their biggest and smallest eigenvalue, namely $\lambda_{M}$ and $\lambda_{m}$, satisfy $\lambda_{m}<1<\lambda_{M}$. Assume further, for simplicity, $\lambda_{m}^{2}+\lambda_{M}^{2}>2$ and $\frac{1}{2} \leq \lambda_{m} \lambda_{M}<1$. We can take for example $\lambda_{m}=0.9$ and $\lambda_{M}=1$.1. It can be computed that $\left(S O(3) \mathrm{U}_{1} \cup S O(3) \mathrm{U}_{2}\right)^{q c}$ coincides with the set of matrices $\mathrm{F}$ satisfying (5.43) $-(5.44)$, that is such that $0<\alpha, \beta \leq \frac{1}{2}\left(\lambda_{m}^{2}+\lambda_{M}^{2}\right)$ and $\alpha \beta \geq \lambda_{m} \lambda_{M}$. Consider now $\mathbf{F}=1+\mathbf{a}(s) \otimes \mathbf{n}$, then

$$
\alpha=1+n_{1}^{2}\left(\mathfrak{D}^{2}-1+s^{2}\right)+s n_{1} n_{2}, \quad \beta=1+n_{2}^{2}\left(\mathfrak{D}^{2}-1+s^{2}\right)-s n_{1} n_{2} .
$$

Choosing for simplicity $n_{2}=0$ and $s$ small enough, we get

$$
0<\alpha, \beta<\frac{1}{2}\left(\lambda_{m}^{2}+\lambda_{M}^{2}\right), \quad \alpha \beta \geq \lambda_{m} \lambda_{M}=\mathfrak{D}^{2} .
$$

Thus, there exists $\varepsilon>0$, and an open interval $[-\varepsilon, \varepsilon]$ such that for every smooth function $f: \mathbb{R} \rightarrow[-\varepsilon, \varepsilon]$ we have that

$$
1+\mathbf{a}\left(f\left(\mathbf{x} \cdot \mathbf{n}^{\perp}\right)\right) \otimes \mathbf{n},
$$

is the gradient of a smooth map $\mathbf{y}$, which is not constant, and which satisfies $\nabla \mathbf{y}(\mathbf{x}) \in\left(S O(3) \mathrm{U}_{1} \cup\right.$ $\left.S O(3) \mathrm{U}_{2}\right)^{q c}$ and $(\underline{\mathrm{H} 1})-(\underline{\mathrm{H} 2})$ for each $\mathbf{x}$. Therefore, a rigidity result as the one in Lemma 5.2 does not hold in general when $\mu=1$.

Remark 5.4. In [16] the author proved that for cubic to monoclinic II phase transitions (and hence also for its special cases of cubic to orthorhombic and cubic to tetragonal phase transitions) necessary and sufficient condition to satisfy $(\mathrm{CC} 1)-(\mathrm{CC} 2)$ with a compound twin is to have $\mu=1$. Is therefore not surprising that the case $\mu=1$ is a special case for Lemma [5.2. This is coherent also with Proposition (5.3) below.

By adding the further hypotheses that $\left.\mathbf{y}\right|_{\partial \Omega}$ is the restriction on $\partial \Omega$ of a $1-1$ map, we can extend Lemma 5.2 to general two well problems

Proposition 5.3. Let $\mathrm{U}, \mathrm{V} \in \mathbb{R}_{\text {Sym }}^{3 \times 3}$ and $\mathrm{R}_{I}, \mathrm{R}_{I I} \in S O(3), \mathbf{b}_{I}, \mathbf{b}_{I I}, \mathbf{m}_{I}, \mathbf{m}_{I I} \in \mathbb{R}^{3} \backslash\{\mathbf{0}\}$ satisfy

$$
\mathrm{R}_{i} \mathrm{~V}=\mathrm{U}+\mathbf{b}_{i} \otimes \mathbf{m}_{i}, \quad i=I, I I,
$$

where $\left(\mathrm{U}, \mathbf{b}_{I}, \mathbf{m}_{I}\right),\left(\mathrm{U}, \mathbf{b}_{I I}, \mathbf{m}_{I I}\right)$ do not fulfil (CC2). Assume $\mathbf{y} \in W^{1, \infty}\left(\Omega ; \mathbb{R}^{3}\right)$ is such that $\left.\mathbf{y}\right|_{\partial \Omega}=$ $\left.\mathbf{y}_{0}\right|_{\partial \Omega}$ for some $\mathbf{y}_{0} \in C\left(\bar{\Omega} ; \mathbb{R}^{3}\right)$ which is $1-1$ in $\Omega$, and

$$
\nabla \mathbf{y}(\mathbf{x}) \in(S O(3) \cup \cup S O(3) \mathrm{V})^{q c}, \quad \nabla \mathbf{y}(\mathbf{x})=1+\mathbf{a}(\mathbf{x}) \otimes \mathbf{n}(\mathbf{x}),
$$

a.e. in $\Omega$, for some $\mathbf{a} \in L^{\infty}\left(\Omega ; \mathbb{R}^{3}\right), \mathbf{n} \in L^{\infty}\left(\Omega ; \mathbb{S}^{2}\right)$. Then,

$$
\nabla \mathbf{y}=\text { constant } \text {. }
$$


Proof. Following the approach devised in [8], we introduce the orthonormal system of coordinates

$$
\mathbf{u}_{1}^{i}:=\frac{\mathrm{U}^{-1} \mathbf{m}_{i}}{\left|\mathbf{U}^{-1} \mathbf{m}_{i}\right|}, \quad \mathbf{u}_{3}^{i}:=\frac{\mathbf{b}_{i}}{\left|\mathbf{b}_{i}\right|}, \quad \mathbf{u}_{2}^{i}:=\mathbf{u}_{3}^{i} \times \mathbf{u}_{1}^{i},
$$

with $i=I, I I$ to be chosen later, and let

$$
\mathrm{L}_{i}:=\mathrm{U}^{-1}\left(1-\delta^{i} \mathbf{u}_{3}^{i} \otimes \mathbf{u}_{1}^{i}\right), \quad \delta^{i}=\frac{1}{2}\left|\mathrm{U}^{-1} \mathbf{m}_{i}\right|\left|\mathbf{b}_{i}\right|
$$

We set $\mathbf{z}^{i}(\mathbf{x}):=\mathbf{y}\left(\mathrm{L}_{i} \mathbf{x}\right)$ and the problem becomes equivalent to find a map $\mathbf{z}^{i} \in W^{1, \infty}\left(\Omega ; \mathbb{R}^{3}\right)$ such that

$$
\nabla \mathbf{z}^{i}(\mathbf{x}) \in\left(S O(3) \mathrm{S}_{i}^{-} \cup S O(3) \mathrm{S}_{i}^{+}\right)^{q c}, \quad \text { a.e. } \mathbf{x} \in \Omega^{L_{i}}:=\left\{\mathbf{x}: \mathrm{L}_{i} \mathbf{x} \in \Omega\right\},
$$

with $\mathbf{S}_{i}^{ \pm}=1 \pm \delta^{i} \mathbf{u}_{3}^{i} \otimes \mathbf{u}_{1}^{i}$, and

$$
\nabla \mathbf{z}^{i}(\mathbf{x})=\mathrm{L}_{i}+\mathbf{a}\left(\mathrm{L}_{i} \mathbf{x}\right) \otimes \mathrm{L}_{i}^{T} \mathbf{n}\left(\mathrm{L}_{i} \mathbf{x}\right), \quad \text { a.e. in } \Omega^{\mathrm{L}_{i}},
$$

where $\mathbf{a} \in L^{\infty}\left(\Omega ; \mathbb{R}^{3}\right), \mathbf{n} \in L^{\infty}\left(\Omega ; \mathbb{S}^{2}\right)$ are as in (5.56). By [7], $\mathbf{z}^{i}$ is a plane strain and satisfies

$$
\mathbf{z}^{i}(\mathbf{x})=\mathbf{Q}\left(z_{1}^{i}\left(s_{1}^{i}, s_{3}^{i}\right) \mathbf{u}_{1}^{i}+s_{2} \mathbf{u}_{2}^{i}+z_{3}^{i}\left(s_{1}^{i}, s_{3}^{i}\right) \mathbf{u}_{3}^{i}\right)
$$

for some $\mathrm{Q} \in S O(3)$, some Lipschitz functions $z_{1}^{i}, z_{2}^{i}$, and where $s_{j}^{i}=\mathbf{x} \cdot \mathbf{u}_{j}^{i}, j=1,2,3$. As a consequence, from (5.58)-(5.59) we deduce

$$
\begin{aligned}
\mathbf{u}_{2}^{i} & =\mathrm{Q}^{T} \nabla \mathbf{z}^{i}(\mathbf{x}) \mathbf{u}_{2}^{i}=\mathrm{Q}^{T} \mathrm{~L}_{i} \mathbf{u}_{2}^{i}+\mathrm{Q}^{T} \mathbf{a}\left(\mathrm{L}_{i} \mathbf{x}\right)\left(\mathbf{n}\left(\mathrm{L}_{i} \mathbf{x}\right) \cdot \mathrm{L}_{i} \mathbf{u}_{2}^{i}\right), \\
\mathbf{u}_{2}^{i} & =\left(\nabla \mathbf{z}^{i}(\mathbf{x})\right)^{T} \mathbf{Q} \mathbf{u}_{2}^{i}=\mathrm{L}_{i}^{T} \mathbf{Q} \mathbf{u}_{2}^{i}+\mathrm{L}_{i}^{T} \mathbf{n}\left(\mathrm{L}_{i} \mathbf{x}\right)\left(\mathbf{a}\left(\mathrm{L}_{i} \mathbf{x}\right) \cdot \mathbf{Q} \mathbf{u}_{2}^{i}\right),
\end{aligned}
$$

a.e. in $\Omega^{\mathrm{L}_{i}}$. That is,

$$
\begin{array}{r}
\mathbf{a}(\mathbf{x})\left(\mathbf{n}(\mathbf{x}) \cdot \mathrm{L}_{i} \mathbf{u}_{2}^{i}\right)=\left(\mathrm{Q}-\mathrm{L}_{i}\right) \mathbf{u}_{2}^{i}, \\
\mathbf{n}(\mathbf{x})\left(\mathbf{a}(\mathbf{x}) \cdot \mathbf{Q} \mathbf{u}_{2}^{i}\right)=\left(\mathrm{L}_{i}^{-T}-\mathrm{Q}\right) \mathbf{u}_{2}^{i},
\end{array}
$$

a.e. in $\Omega$. Let us now consider the function

$$
f_{i}(\mu)=\operatorname{det}\left(\left(\mathrm{U}+\mu \mathbf{b}_{i} \otimes \mathbf{m}_{i}\right)^{T}\left(\mathrm{U}+\mu \mathbf{b}_{i} \otimes \mathbf{m}_{i}\right)-1\right), \quad \mu \in[0,1], i=I, I I .
$$

Thanks to [6, Prop. 5] we know that $f_{i}$ is a quadratic polynomial and $f_{i}(\mu)=f_{i}(1-\mu)$. We first notice that

$$
\begin{aligned}
f_{i}(\mu) & =(\operatorname{det} \mathrm{U}) \operatorname{det}\left(\left(\mathrm{U}+\mu \mathbf{b}_{i} \otimes \mathbf{m}_{i}\right)-\left(\mathrm{U}+\mu \mathbf{b}_{i} \otimes \mathbf{m}_{i}\right)^{-T}\right) \\
& =\left(\operatorname{det} \mathrm{U}^{2}\right) \operatorname{det}\left(\left(1-\mathrm{U}^{-2}\right)+\mu 2 \delta\left(\mathbf{u}_{3}^{i} \otimes \mathbf{u}_{1}^{i}+\mathbf{u}_{1}^{i} \otimes \mathrm{U}^{-2} \mathbf{u}_{3}^{i}\right)\right) \\
& =\operatorname{det}\left(\left(\mathrm{U}^{2}-1\right)+\mu 2 \delta\left(\mathbf{u}_{3}^{i} \otimes \mathrm{U}^{2} \mathbf{u}_{1}^{i}+\mathbf{u}_{1}^{i} \otimes \mathbf{u}_{3}^{i}\right)\right)
\end{aligned}
$$

A derivation of $f_{i}$ with respect to $\mu$ leads

$$
\begin{aligned}
& f_{i}^{\prime}(\mu) \\
& =2 \delta\left(\operatorname{det} \mathrm{U}^{2}\right) \operatorname{cof}\left(\left(1-\mathrm{U}^{-2}\right)+\mu 2 \delta\left(\mathbf{u}_{3}^{i} \otimes \mathbf{u}_{1}^{i}+\mathbf{u}_{1}^{i} \otimes \mathrm{U}^{-2} \mathbf{u}_{3}^{i}\right)\right):\left(\mathbf{u}_{3}^{i} \otimes \mathbf{u}_{1}^{i}+\mathbf{u}_{1}^{i} \otimes \mathrm{U}^{-2} \mathbf{u}_{3}^{i}\right) \\
& =2 \delta\left(\operatorname{det} \mathrm{U}^{2}\right)\left(\operatorname{cof}\left(1-\mathrm{U}^{-2}\right) \mathbf{u}_{1}^{i} \cdot\left(\mathbf{u}_{3}^{i}+\mathrm{U}^{-2} \mathbf{u}_{3}^{i}\right)+\mu 4 \delta\left(1-\mathrm{U}^{-2}\right) \mathbf{u}_{2}^{i} \cdot\left(\mathbf{u}_{1}^{i} \times \mathrm{U}^{-2} \mathbf{u}_{3}^{i}\right)\right) \\
& =2 \delta\left(\operatorname{cof}\left(\mathrm{U}^{2}-1\right) \mathbf{u}_{3}^{i} \cdot\left(\mathbf{u}_{1}^{i}+\mathrm{U}^{2} \mathbf{u}_{1}^{i}\right)+\mu 4 \delta\left(\mathrm{U}^{2}-1\right) \mathbf{u}_{2}^{i} \cdot\left(\mathrm{U}^{2} \mathbf{u}_{1}^{i} \times \mathbf{u}_{3}^{i}\right)\right) .
\end{aligned}
$$


Here we made use of the fact that $\operatorname{cof}\left(\sum_{i} \mathbf{v}_{i} \otimes \mathbf{w}_{i}\right)=\sum_{i<j} \mathbf{v}_{i} \times \mathbf{v}_{j} \otimes \mathbf{w}_{i} \times \mathbf{w}_{j}$. We now fix $i=I$ and claim that, under our hypotheses, there exist no $\mathrm{Q} \in S O(3)$ such that $\left(\mathrm{Q}-\mathrm{L}_{I}^{-T}\right) \mathbf{u}_{2}^{I}=\mathbf{0}$. This, by (5.61) and the fact that $|\mathbf{n}|=1$ a.e. implies that $\mathbf{n}$ is, up to a change of sign, equal to a constant. That is, $\mathbf{n} \operatorname{sgn}\left(n_{j}\right)$ is constant, for some $j=1,2,3$ such that $\left|n_{j}\right| \neq 0$ a.e. in $\Omega$. To prove the claim we argue by contradiction, and notice that the existence of $\mathrm{Q} \in S O(3)$ satisfying $\left(\mathrm{Q}-\mathrm{L}_{I}^{-T}\right) \mathbf{u}_{2}^{I}=\mathbf{0}$ implies $\left|\mathrm{L}_{I}^{-T} \mathbf{u}_{2}^{I}\right|=1$, that is

$$
\left(\mathrm{U}^{2}-1\right) \mathbf{u}_{2}^{I} \cdot \mathbf{u}_{2}^{I}=0
$$

Let us notice now that

$$
\left(\mathrm{U}^{2} \mathbf{u}_{1}^{I} \times \mathbf{u}_{3}^{I}\right) \times \mathbf{u}_{2}^{I}=\left(\mathrm{U}^{2} \mathbf{u}_{1}^{I} \cdot\left(\mathbf{u}_{1}^{I} \times \mathbf{u}_{3}^{I}\right)\right) \mathbf{u}_{3}^{I} .
$$

By making use of (2.8) in (5.63) we show that $\mathrm{U}^{2} \mathbf{u}_{1}^{I} \cdot\left(\mathbf{u}_{1}^{I} \times \mathbf{u}_{3}^{I}\right)=0$, which by (5.63) implies that $\mathrm{U}^{2} \mathbf{u}_{1}^{I} \times \mathbf{u}_{3}^{I}$ is parallel to $\mathbf{u}_{2}^{I}$. Therefore, by (5.62), we get that $f_{I}^{\prime}$ is constant in $\mu$. Furthermore, as $f_{I}$ is a quadratic polynomial and $f_{I}(\mu)=f_{I}(1-\mu)$ we have that $f_{I}^{\prime}\left(\frac{1}{2}\right)=0$ and hence $f_{I}^{\prime}$ is identically 0 . But, as

$$
\left.f_{I}^{\prime}(\mu)\right|_{\mu=0}=2 \mathbf{b}_{I} \cdot \mathrm{U} \operatorname{cof}\left(\mathrm{U}^{2}-1\right) \mathbf{m}_{I}=0,
$$

we contradict the assumption that $\left(\mathbf{U}, \mathbf{b}_{I}, \mathbf{m}_{I}\right)$ does not satisfy $(\mathrm{CC} 2)$ concluding the proof of the claim. We now fix $i=I I$ and claim that, under our hypotheses, there exist no $\mathrm{Q} \in S O(3)$ such that $\left(\mathrm{Q}-\mathrm{L}_{I I}\right) \mathbf{u}_{2}^{I I}=\mathbf{0}$. This, by (5.60) and the fact that $\operatorname{sgn}\left(n_{j}\right) \mathbf{n}$ is a constant implies that $\operatorname{sgn}\left(n_{j}\right) \mathbf{a}$ is also a constant. To prove the claim we argue again by contradiction, and notice that the existence of $\mathrm{Q} \in S O(3)$ satisfying $\left(\mathrm{Q}-\mathrm{L}_{I I}\right) \mathbf{u}_{2}^{I I}=\mathbf{0}$ implies $\left|\mathrm{L}_{I I} \mathbf{u}_{2}^{I I}\right|=1$, and thus

$$
\left(\mathrm{U}^{-2}-1\right) \mathbf{u}_{2}^{I I} \cdot \mathbf{u}_{2}^{I I}=0 .
$$

By making use of (2.9) we can now show that $\mathbf{u}_{1}^{I I} \times \mathrm{U}^{-2} \mathbf{u}_{3}^{I I}$ is parallel to $\mathbf{u}_{2}^{I I}$, and hence, by (5.64), that $f_{I I}^{\prime}$ is constant in $\mu$ and identically 0 . But, noticing that

$$
\left.f_{I I}^{\prime}(\mu)\right|_{\mu=0}=2 \mathbf{b}_{I I} \cdot \mathrm{U} \operatorname{cof}\left(\mathrm{U}^{2}-1\right) \mathbf{m}_{I I}=0,
$$

we contradict the assumption that $\left(\mathbf{U}, \mathbf{b}_{I I}, \mathbf{m}_{I I}\right)$ does not satisfy $(\mathrm{CC} 2)$ concluding the proof of the second claim.

In conclusion, we proved that $\operatorname{sgn}\left(n_{j}\right) \mathbf{n}$ and $\operatorname{sgn}\left(n_{j}\right) \mathbf{a}$ are constants, and therefore so must be $\nabla \mathbf{y}$.

Remark 5.5. It is clear from the proof of Proposition 5.3 that, if the type I solution $\left(\mathrm{U}, \mathbf{b}_{I}, \mathbf{m}_{I}\right)$ of the twinning equation (2.6) does not satisfy (CC2), but the type II solution $\left(\mathbf{U}, \mathbf{b}_{I I}, \mathbf{m}_{I I}\right)$ of (2.6) does, then we can guarantee that $\mathbf{n}$ in (5.56) is constant up to a change of sign. Similarly, if the type I solution $\left(\mathrm{U}, \mathbf{b}_{I}, \mathbf{m}_{I}\right)$ of the twinning equation (2.6) does satisfy (CC2), but the type II solution $\left(\mathrm{U}, \mathbf{b}_{I I}, \mathbf{m}_{I I}\right)$ of (2.6) does not, then the direction of $\mathbf{a}$ in (5.56) is constant. That is, there exists $\mathbf{v} \in \mathbb{R}^{3}$ such that $\mathbf{a} \times \mathbf{v}=0$ a.e. in $\Omega$. We refer the reader to Proposition 6.1 and Proposition 6.2 for examples of non-affine maps when (CC2) is not satisfied.

\section{Macroscopic moving interfaces}

In this section, we use the theory of the previous sections to prove some results about moving interfaces in martensitic transformations. The results are different for different type of twins. We start 
with compound twins and we recall that, by Proposition 2.3 , two martensite variants $\mathrm{U}_{1}, \mathrm{U}_{2} \in \mathbb{R}_{S y m^{+}}^{3 \times 3}$ form a compound twin if and only if there exist $\mu>0, \mathbf{v} \in \mathbb{S}^{2}$ such that $\mathrm{U}_{1} \mathbf{v}=\mathrm{U}_{2} \mathbf{v}=\mu \mathbf{v}$. Thanks to Lemma 5.2 we can prove that in this case moving interfaces need to be planar and the related macroscopic gradient constant.

Theorem 6.1. Let $\mathrm{U}_{1}, \mathrm{U}_{2} \in \mathbb{R}_{\text {Sym }}^{3 \times 3}$ be a compound twin and such that

$$
\mathrm{U}_{1} \mathbf{w}=\mathrm{U}_{2} \mathbf{w}=\mu \mathbf{w}
$$

for some $\mathbf{w} \in \mathbb{R}^{3}, \mu \neq 1$. Then, every $\mathbf{y}$ satisfying the regular moving mask approximation and such that

$$
\nabla \mathbf{y} \in\left(S O(3) \mathrm{U}_{1} \cup S O(3) \mathrm{U}_{2}\right)^{q c}, \quad \text { a.e. in } \Omega
$$

is constant, and the related moving interfaces planar.

Proof. As y satisfies a regular moving mask approximation, by Theorem 4.1 we know that $\nabla \mathbf{y}=$ $1+\mathbf{a} \otimes \mathbf{n}$ for some $\mathbf{a} \in L^{\infty}\left(\Omega ; \mathbb{R}^{3}\right), \mathbf{n} \in L^{\infty}\left(\Omega ; \mathbb{S}^{2}\right)$. Since $\mu \neq 1$, we can apply Lemma 5.2 and thus deduce that $\nabla \mathbf{y}$ is constant in $\Omega$. The function $\mathbf{z}(\mathbf{x}):=\mathbf{y}(\mathbf{x})-\mathbf{x}$ is such that $\mathbf{z} \in W^{1, \infty}\left(\Omega ; \mathbb{R}^{3}\right)$ and is constant in every connected component of $\Omega_{A}(t)$, for each $t \geq 0$. Thus, $\Gamma(t)$ must be a (or at least the union of disconnected subsets of a) level-set for $\mathbf{z}$, and hence a plane (or union of disconnected planes) as $\nabla \mathbf{z}$ is constant and rank-one in $\Omega$.

By arguing in the same way, Theorem 6.1 can be generalised to a wider range of situations as stated in Theorem 6.2 below. This is relevant, for example, in the cubic to monoclinic transformation occurring in $\mathrm{Zn}_{45} \mathrm{Au}_{30} \mathrm{Cu}_{25}$, where there are 3 sets of four deformation gradients satisfying the hypotheses of Theorem 6.2 .

Theorem 6.2. Let $\mathrm{U}_{1}, \ldots, \mathrm{U}_{N} \in \mathbb{R}_{\text {Sym }}^{3 \times 3}$ be such that $\mathrm{U}_{i} \mathbf{w}=\mu \mathbf{w}$ and $\operatorname{det} \mathrm{U}_{i}=\mathfrak{D}$ for some $\mathbf{w} \in \mathbb{R}^{3}$, $\mu \neq 1$ and every $i=1, \ldots, n$. Then, every $\mathbf{y}$ satisfying the regular moving mask approximation and such that

$$
\nabla \mathbf{y} \in\left(\cup_{i=1}^{N} S O(3) \cup_{i}\right)^{q c}, \quad \text { a.e. in } \Omega
$$

is constant, and the related moving interfaces planar.

An equivalent result can be proved, in the same way, for the general two well problem under the additional assumption that $\mathbf{y}$ coincides on $\partial \Omega$ with a $1-1$ map.

Theorem 6.3. Let $\mathrm{U}, \mathrm{\vee} \in \mathbb{R}_{\text {Sym }}^{3 \times 3}$ and $\mathrm{R}_{I}, \mathrm{R}_{I I} \in S O(3), \mathbf{b}_{I}, \mathbf{b}_{I I}, \mathbf{m}_{I}, \mathbf{m}_{I I} \in \mathbb{R}^{3} \backslash\{\mathbf{0}\}$ satisfy

$$
\mathrm{R}_{i} \mathrm{~V}=\mathrm{U}+\mathbf{b}_{i} \otimes \mathbf{m}_{i}, \quad i=I, I I,
$$

where $\left(\mathrm{U}, \mathbf{b}_{I}, \mathbf{m}_{I}\right),\left(\mathrm{U}, \mathbf{b}_{I I}, \mathbf{m}_{I I}\right)$ do not fulfil (CC2). Then, every $\mathbf{y}$ satisfying the regular moving mask approximation, such that $\left.\mathbf{y}\right|_{\partial \Omega}=\left.\mathbf{y}_{0}\right|_{\partial \Omega}$ for some $\mathbf{y}_{0} \in C\left(\bar{\Omega} ; \mathbb{R}^{3}\right)$ which is $1-1$ in $\Omega$, and such that

$$
\nabla \mathbf{y}(\mathbf{x}) \in(S O(3) \cup \cup S O(3) \mathrm{V})^{q c},
$$

is constant, and the related moving interfaces planar. 
The next result is related to type I twins satisfying the cofactor conditions. In this case we can prove that simple laminates can form macroscopically curved families of austenite-martensite interfaces with no transition layer. The proof strongly relies on Theorem 2.2 .

Proposition 6.1. Let $\mathrm{U}_{1}$ and $\mathrm{U}_{2}$ be two martensitic variants and $\hat{\mathrm{R}} \in S O(3), \mathbf{b}_{I}, \mathbf{m}_{I} \in \mathbb{R}^{3}$ be a type I solution to (2.6) and satisfying the cofactor conditions. Then, there exist $\mathrm{R}_{0}, \mathrm{R}_{1} \in S O(3), \xi \in \mathbb{R}$, $\mathbf{a}_{0} \in \mathbb{R}^{3}, \mathbf{n}_{0}, \mathbf{n}_{1} \in \mathbb{S}^{2}$ such that for every $\lambda \in L^{\infty}(\Omega ;[0,1])$ satisfying $\nabla \lambda \times\left(\xi \mathbf{n}_{1}-\mathbf{n}_{0}\right)=\mathbf{0}$ in the sense of distributions, there exists $\mathbf{y} \in W^{1, \infty}\left(\Omega ; \mathbb{R}^{3}\right)$ with

$$
\nabla \mathbf{y}=\mathrm{R}_{0}\left[(1-\lambda) \mathrm{U}_{1}+\lambda \hat{\mathrm{R}} \mathrm{U}_{2}\right]=1+\mathbf{a}_{0} \otimes\left(\lambda \xi \mathbf{n}_{1}+(1-\lambda) \mathbf{n}_{0}\right), \quad \text { a.e. in } \Omega .
$$

Furthermore, $\mathbf{y}$ satisfies a regular moving mask approximation, the related moving interfaces are curved, and $\nabla \cdot\left(\left|\lambda \xi \mathbf{n}_{1}+(1-\lambda) \mathbf{n}_{0}\right| \mathbf{a}_{0}\right)=0$ in the sense of distributions.

Proof. From Theorem 2.2 and in particular from (2.12) we know the existence of $\mathrm{R}_{0}, \mathrm{R}_{1} \in S O(3)$, $\xi \in \mathbb{R}, \mathbf{a}_{0} \in \mathbb{R}^{3}, \mathbf{n}_{0}, \mathbf{n}_{1} \in \mathbb{S}^{2}$ such that

$$
\mathrm{R}_{0}\left[(1-\lambda) \mathrm{U}_{1}+\lambda \hat{\mathrm{R}} \mathrm{U}_{2}\right]=1+\mathbf{a}_{0} \otimes\left(\lambda \xi \mathbf{n}_{1}+(1-\lambda) \mathbf{n}_{0}\right), \quad \text { for all } \lambda \in[0,1] .
$$

We thus choose $\lambda \in L^{\infty}(\Omega)$ to be a function such that $\lambda \in[0,1]$ a.e., and define

$$
\mathbf{a}(\mathbf{x}):=\mathbf{a}_{0}\left|\mathbf{n}_{0}+\lambda(\mathbf{x})\left(\xi \mathbf{n}_{1}-\mathbf{n}_{0}\right)\right|, \quad \mathbf{n}(\mathbf{x}):=\frac{\mathbf{n}_{0}+\lambda(\mathbf{x})\left(\xi \mathbf{n}_{1}-\mathbf{n}_{0}\right)}{\left|\mathbf{n}_{0}+\lambda\left(\xi \mathbf{n}_{1}-\mathbf{n}_{0}\right)\right|}
$$

so that $\mathbf{n}$ has unitary norm. In the notation of Theorem 2.2, we have

$$
\operatorname{det}\left(\mathrm{R}_{0}\left[(1-\lambda) \mathrm{U}_{1}+\lambda \hat{\mathrm{R}} \mathrm{U}_{2}\right]\right)=\operatorname{det}\left(\mathrm{R}_{0}\right) \operatorname{det}\left(\mathrm{U}_{1}+\lambda \mathbf{b}_{I} \otimes \mathbf{m}_{I}\right)=\operatorname{det} \mathrm{U}_{1}
$$

where the Sherman-Morrison inversion formula and the fact that $\mathrm{U}_{1}^{-1} \mathbf{n} \cdot \mathbf{a}=0$ have been used. That is,

$$
\mathbf{a}_{0} \cdot\left(\mathbf{n}_{0}+\lambda\left(\xi \mathbf{n}_{1}-\mathbf{n}_{0}\right)\right)
$$

is constant independently of $\lambda$, or, in an equivalent way,

$$
\mathbf{a}_{0} \cdot\left(\xi \mathbf{n}_{1}-\mathbf{n}_{0}\right)=0
$$

We just need to check if it is possible to have

$$
\nabla \times\left(a_{i} \mathbf{n}\right)=0
$$

Exploiting the definition of $\mathbf{a}$ and $\mathbf{n}$ get that this is satisfied if and only if

$$
\nabla \lambda \times\left(\xi \mathbf{n}_{1}-\mathbf{n}_{0}\right)=\mathbf{0},
$$

in a weak sense. Therefore, if $\Omega$ is convex $\lambda$ must satisfy $\lambda(\mathbf{x})=f\left(\mathbf{x} \cdot\left(\xi \mathbf{n}_{1}-\mathbf{n}_{0}\right)\right)$, for some $f \in$ $L^{\infty}(\mathbb{R} ;[0,1])$, and thus

$$
\mathbf{y}=\mathbf{x}+\mathbf{a}_{0}\left(\mathbf{n}_{0} \cdot \mathbf{x}+F\left(\mathbf{x} \cdot\left(\xi \mathbf{n}_{1}-\mathbf{n}_{0}\right)\right)+\mathbf{c},\right.
$$

for some constant $\mathbf{c} \in \mathbb{R}^{3}$, and where $F(s)=\int_{0}^{s} f(s) \mathrm{d} s$. Therefore, after choosing

$$
I_{T}:=\left(\inf _{\mathbf{x} \in \Omega} G(\mathbf{x}), \sup _{\mathbf{x} \in \Omega} G(\mathbf{x})\right),
$$


where $G(\mathbf{x})=\mathbf{n}_{0} \cdot \mathbf{x}+F\left(\mathbf{x} \cdot\left(\xi \mathbf{n}_{1}-\mathbf{n}_{0}\right)\right)$, we deduce that the image of $\mathbf{z}(\mathbf{x})=\mathbf{y}(\mathbf{x})-\mathbf{x}$ is $\mathbf{c}+t \mathbf{a}_{0}$, for $t \in I_{T}$. If $\Omega$ is connected but not convex, then $\lambda$ might not be of the form $f\left(\mathbf{x} \cdot\left(\xi \mathbf{n}_{1}-\mathbf{n}_{0}\right)\right)$, but the image of $\mathbf{z}(\mathbf{x})=\mathbf{y}(\mathbf{x})-\mathbf{x}$ is still contained in the one-dimensional line $\mathbf{c}+t \mathbf{a}_{0}$, for some $\mathbf{c} \in \mathbb{R}^{3}$ and $t$ in some bounded interval $\hat{I}_{T}$. We can thus use Corollary 4.1 and deduce the existence of a family of moving interfaces, which are also level sets for $\mathbf{z}(\mathbf{x})$.

In order to prove that $\nabla \cdot \mathbf{a}$, we first mollify $\lambda$ and, defined $\mathbf{m}$ as $\mathbf{m}:=\xi \mathbf{n}_{1}-\mathbf{n}_{0}$, notice that thanks to Fubini's theorem for distributions we can write

$$
\left\langle\nabla \lambda_{\varepsilon} \times \mathbf{m}, \boldsymbol{\psi}\right\rangle_{\mathcal{D}^{\prime}, \mathcal{D}}=\left\langle\nabla \lambda,\left(\mathbf{m} \times \boldsymbol{\psi}_{\varepsilon}\right)\right\rangle_{\mathcal{D}^{\prime}, \mathcal{D}}=\left\langle\nabla \lambda \times \mathbf{m}, \boldsymbol{\psi}_{\varepsilon}\right\rangle_{\mathcal{D}^{\prime}, \mathcal{D}}=0,
$$

thanks to (6.66), for all $\psi \in C_{c}^{\infty}\left(\Omega, \mathbb{R}^{3}\right)$. Therefore, $\nabla \lambda_{\varepsilon} \| \mathbf{m}$, and, by (6.65),

$$
\nabla \lambda_{\varepsilon} \cdot \mathbf{a}_{0}=0 .
$$

On the other hand, exploiting the smooth dependence on $\lambda$ of $\mathbf{a}$ and the fact that $\lambda_{\varepsilon} \rightarrow \lambda$ in $L^{p}(\Omega)$ for every $p \in[1, \infty)$, we have

$$
\int_{\Omega} \mathbf{a}(\lambda) \cdot \nabla \psi \mathrm{d} \mathbf{x}=\lim _{\varepsilon \rightarrow 0} \int_{\Omega} \mathbf{a}\left(\lambda_{\varepsilon}\right) \cdot \nabla \psi \mathrm{d} \mathbf{x}=-\lim _{\varepsilon \rightarrow 0} \int_{\Omega} g^{\prime}\left(\lambda_{\varepsilon}\right) \mathbf{a}_{0} \cdot \nabla \lambda_{\varepsilon} \psi \mathrm{d} \mathbf{x}
$$

for every $\psi \in C_{c}^{\infty}(\Omega)$ and where $g(s)=\left|\mathbf{n}_{0}+s\left(\xi \mathbf{n}_{1}-\mathbf{n}_{0}\right)\right|$. The last term in the chain of identities above is null due to (6.67), and therefore the proof is concluded.

Finally, a result related to type II twins satisfying the cofactor conditions:

Proposition 6.2. Let $\mathrm{U}_{1}$ and $\mathrm{U}_{2}$ be two martensitic variants and $\hat{\mathrm{R}} \in S O(3), \mathbf{b}_{I I}, \mathbf{m}_{I I} \in \mathbb{R}^{3}$ be a type II solution to (2.6) and satisfying the cofactor conditions. Then, there exist $\mathrm{R}_{0} \in S O(3), \xi \in \mathbb{R}$, $\mathbf{a}_{0}, \mathbf{a}_{1} \in \mathbb{R}^{3}, \mathbf{n}_{0} \in \mathbb{S}^{2}$ such that for every $\lambda \in L^{\infty}(\Omega ;[0,1])$ satisfying $\nabla \lambda \times \mathbf{n}_{0}=\mathbf{0}$ in the sense of distributions, there exists $\mathbf{y} \in W^{1, \infty}\left(\Omega ; \mathbb{R}^{3}\right)$ with

$$
\nabla \mathbf{y}=\mathrm{R}_{0}\left[(1-\lambda) \mathrm{U}_{1}+\lambda \hat{\mathrm{R}} \mathrm{U}_{2}\right]=1+\left(\lambda \xi \mathbf{a}_{1}+(1-\lambda) \mathbf{a}_{0}\right) \otimes \mathbf{n}_{0}, \quad \text { a.e. in } \Omega .
$$

Furthermore, y satisfies a regular moving mask approximation, and

$$
\nabla \cdot\left(\lambda \xi \mathbf{a}_{1}+(1-\lambda) \mathbf{a}_{0}\right)=0
$$

in the sense of distributions.

Proof. From Theorem (2.3) and in particular from (2.14) we know the existence of $\mathrm{R}_{0}, \mathrm{R}_{1} \in S O(3)$, $\xi \in \mathbb{R}, \mathbf{a}_{0}, \mathbf{a}_{1} \in \mathbb{R}^{3}, \mathbf{n}_{0} \in \mathbb{S}^{2}$ satisfying

$$
\mathrm{R}_{0}\left[(1-\lambda) \mathrm{U}_{1}+\lambda \hat{\mathrm{R}} \mathrm{U}_{2}\right]=1+\left(\lambda \xi \mathbf{a}_{1}+(1-\lambda) \mathbf{a}_{0}\right) \otimes \mathbf{n}_{0}, \quad \text { for all } \lambda \in[0,1] .
$$

Thus choose $\lambda \in L^{\infty}(\Omega)$ to be a function such that $\lambda \in[0,1]$ a.e., and define

$$
\mathbf{a}(\mathbf{x}):=\left(\mathbf{a}_{0}+\lambda(\mathbf{x})\left(\xi \mathbf{a}_{1}-\mathbf{a}_{0}\right)\right)
$$

It is trivial to check, by arguing as in the proof of Proposition 6.1, that also (H2) holds.

Now, by taking the curl of $\nabla \mathbf{y}_{i}$ we deduce that

$$
\nabla \times\left(\lambda \mathbf{n}_{0}\right)=\nabla \lambda \times \mathbf{n}_{0}=0
$$

in the sense of distributions. Therefore taking $\lambda$ such that $\nabla \lambda \| \mathbf{n}$ in a weak sense, by Proposition 4.1 and Remark 4.1 follows the existence of a family of moving planar interfaces. The fact that $\nabla \cdot \mathbf{a}=0$ in the sense of distributions trivially follows from Proposition 5.2 . 


\section{$7 \quad$ Experimental evidence}

The physical assumptions which were made in this work and some of the properties that have been deduced here are currently being investigated from an experimental perspective. Indeed, the authors of [13] have used X-ray Laue microdiffraction to measure the orientations and structural parameters of variants and phases in $\mathrm{Zn}_{45} \mathrm{Au}_{30} \mathrm{Cu}_{25}$. With this modern technique, the scanned area is meshed with small rectangles (e.g., in [13] authors use $2 \mu \mathrm{m}$ wide squares), and one can identify the phase and variant in each cuboid which has as a basis a rectangle of the mesh and depth of approximate $2 \mu \mathrm{m}$ from the sample surface. In cubes where a single phase or variant has been recognised, one can also measure the lattice parameters necessary to compute the average deformation gradient. In this way one can investigate what is happening at the phase interface by studying the lattice parameters in mesh rectangles where a martensite variant has been recognised and which have at least one neighbouring rectangle where the Laue microdiffraction was able to identify austenite.

In this way, the authors of [13] compute in some of the mesh cubes lying on the interface the number $\|\operatorname{cof}(\nabla \mathbf{y}-1)\|$, which, as it is easy to verify, is zero if and only if $\nabla \mathbf{y}-1$ is rank-one. Experimental results give $\|\operatorname{cof}(\nabla \mathbf{y}-1)\|$ to be of the order of $10^{-4}$, which seems small enough to be considered zero, and hence to justify (H1).

Further investigations are ongoing to verify that $\nabla \mathbf{y}(\mathbf{x})$ remains constant in time when $\mathbf{x}$ is not on the interface. This seems a reasonable assumption, as long as no external force acts on the sample and as long as one neglects other internal stresses giving rise to elastic deformations which, anyway, seem to be small compared to the deformations induced by the phase transition.

In conclusion, the data collected up till now seem to confirm the validity of the assumptions that we made in the present work. However, in the images in [13] there are many mesh cubes close to some of the phase interfaces where the X-ray Laue microdiffraction is not able to recognize any single variant or phase, and hence where the validity of the assumptions to get (H1) could be questioned or should be verified in some other way.

\section{References}

[1] G. Alberti, S. Bianchini, and G. Crippa. Structure of level sets and Sard-type properties of Lipschitz maps. Ann. Sc. Norm. Super. Pisa Cl. Sci. (5), 12(4):863-902, 2013.

[2] L. Ambrosio, N. Fusco, and D. Pallara. Functions of bounded variation and free discontinuity problems. Oxford Mathematical Monographs. The Clarendon Press, Oxford University Press, New York, 2000.

[3] J.M. Ball and C. Carstensen. Hadamard's compatibility condition for microstructures. In progress.

[4] J.M. Ball and C. Carstensen. Nonclassical austenite-martensite interfaces. Le Journal de Physique IV, 7(C5):C5-35, 1997.

[5] J.M. Ball and C. Carstensen. Compatibility conditions for microstructures and the austenitemartensite transition. Materials Science and Engineering: A, 273:231-236, 1999. 
[6] J.M. Ball and R.D. James. Fine phase mixtures as minimizers of energy. Arch. Rational Mech. Anal., 100(1):13-52, 1987.

[7] J.M. Ball and R.D. James. A characterization of plane strain. Proc. Roy. Soc. London Ser. A, 432(1884):93-99, 1991.

[8] J.M. Ball and R.D. James. Proposed experimental tests of a theory of fine microstructure and the two-well problem. Phil. Trans. R. Soc. Lond. A, 338(1650):389-450, 1992.

[9] J.M. Ball and K. Koumatos. An investigation of non-planar austenite-martensite interfaces. Math. Models Methods Appl. Sci., 24(10):1937-1956, 2014.

[10] K. Bhattacharya. Self-accommodation in martensite. Arch. Rational Mech. Anal., 120(3):201244, 1992.

[11] K. Bhattacharya. Microstructure of martensite: why it forms and how it gives rise to the shapememory effect, volume 2. Oxford University Press, 2003.

[12] X. Chen, V. Srivastava, V. Dabade, and R.D. James. Study of the cofactor conditions: conditions of supercompatibility between phases. J. Mech. Phys. Solids, 61(12):2566-2587, 2013.

[13] X. Chen, N. Tamura, A. MacDowell, and R.D. James. In-situ characterization of highly reversible phase transformation by synchrotron x-ray laue microdiffraction. Applied Physics Letters, 108(21):211902, 2016.

[14] C. Chluba, W. Ge, R.L. de Miranda, J. Strobel, L. Kienle, E. Quandt, and M. Wuttig. Ultralowfatigue shape memory alloy films. Science, 348(6238):1004-1007, 2015.

[15] F. Della Porta. A model for the evolution of highly reversible martensitic transformations. arXiv:1811.07664.

[16] F. Della Porta. On the cofactor conditions and further conditions of supercompatibility between phases. Journal of the Mechanics and Physics of Solids, 122:27 - 53, 2019.

[17] G. Dolzmann. Variational methods for crystalline microstructure-analysis and computation, volume 1803 of Lecture Notes in Mathematics. Springer-Verlag, Berlin, 2003.

[18] G. Dolzmann and S. Müller. Microstructures with finite surface energy: the two-well problem. Arch. Rational Mech. Anal., 132(2):101-141, 1995.

[19] L.C. Evans. Partial differential equations, volume 19 of Graduate Studies in Mathematics. American Mathematical Society, Providence, RI, second edition, 2010.

[20] L.C. Evans and R.F. Gariepy. Measure theory and fine properties of functions. Studies in Advanced Mathematics. CRC Press, Boca Raton, FL, 1992.

[21] H. Federer. Geometric measure theory. Die Grundlehren der mathematischen Wissenschaften, Band 153. Springer-Verlag New York Inc., New York, 1969.

[22] H. Flanders. Differentiation under the integral sign. Amer. Math. Monthly, 80:615-627; correction, ibid. 81 (1974), 145, 1973. 
[23] V. Girault and P.A. Raviart. Finite element approximation of the Navier-Stokes equations, volume 749 of Lecture Notes in Mathematics. Springer-Verlag, Berlin-New York, 1979.

[24] H. Halkin. Implicit functions and optimization problems without continuous differentiability of the data. SIAM J. Control, 12:229-236, 1974. Collection of articles dedicated to the memory of Lucien W. Neustadt.

[25] T. Iwaniec, G.C. Verchota, and A.L. Vogel. The failure of rank-one connections. Arch. Ration. Mech. Anal., 163(2):125-169, 2002.

[26] R.D. James. Taming the temperamental metal transformation. Science, 348(6238):968-969, 2015.

[27] R.D. James. Materials from mathematics. http://www.ams.org/CEB-2018-Master.pdf, 2018.

[28] F. Morgan. Geometric measure theory. Academic Press, Inc., San Diego, CA, second edition, 1995. A beginner's guide.

[29] S. Müller. Variational models for microstructure and phase transitions. In Calculus of variations and geometric evolution problems (Cetraro, 1996), volume 1713 of Lecture Notes in Math., pages 85-210. Springer, Berlin, 1999.

[30] P. Pedregal. Parametrized measures and variational principles, volume 30 of Progress in Nonlinear Differential Equations and their Applications. Birkhäuser Verlag, Basel, 1997.

[31] Y. Song, X. Chen, V. Dabade, T.W. Shield, and R.D. James. Enhanced reversibility and unusual microstructure of a phase-transforming material. Nature, 502(7469):85, 2013.

[32] M.S. Wechsler, D.S. Lieberman, and T.A. Read. On the theory of the formation of martensite. Trans Aime, 197:1503-1515, 1953.

[33] Z. Zhang, R.D. James, and S. Müller. Energy barriers and hysteresis in martensitic phase transformations. Acta Materialia, 57(15):4332-4352, 2009. 